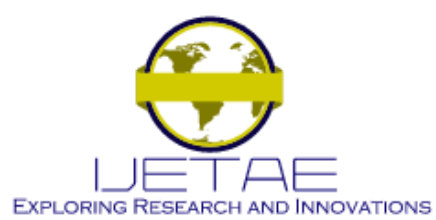

International Journal of Emerging Technology and Advanced Engineering

Website: www.ijetae.com (ISSN 2250-2459, ISO 9001:2008 Certified Journal, Volume 10, Issue 08, August 2020)

\title{
Control System for Application in Residential Environment Using Bluetooth 5
}

\author{
Jorge Luis Bianchetti Junior ${ }^{1}$, Rodrigo Reina Muñoz ${ }^{2}$ \\ ${ }^{1,2}$ Center for Engineering, Modeling and Applied Social Sciences (CECS), Federal University of ABC - UFABC, Santo André, \\ Brazil, S.P. Brazil
}

\begin{abstract}
In the last few decades, there has been an increase in the use of digital devices by the people as a way of facilitate their day-to-day tasks. Several new devices have been developed for the control and automation of residential environments, thus emerging the smart homes concept that is strongly linked to the Internet of Things. Such devices are used for safety, decoration, child care, domestic management, among many other applications, and are implemented using many wireless communication technologies, data storage, sensors and actuators. In this scenario, Bluetooth low energy technology has played important role. Recently, Bluetooth 5.0 emerged with significant improvements, including twice the speed and four times the range compared to the previous generation. Also, it is possible to keep the Bluetooth on for longer time, since consumes less energy than generation 4.0. This enhanced characteristic has stimulated the use of this technology in home automation applications, and so, we explore this technology in this work. This work is aimed to develop a home automation system, consisting of a central hub and two peripheral modules, being them a Smart Power Plug and a LPG Sensor. The communication between the devices was developed using the Bluetooth $^{\circledR} 5.0$ communication protocol and the data were stored in a real-time database of Firebase $^{\circledR}$, which also communicates with an Android application. For this purpose, a NodeMCU ${ }^{\circledR}$ development kit and some boards containing the CC2640R2F microcontroller were used. The results obtained so far, are quite promising to be applied in home automation applications.
\end{abstract}

Keywords - Home automation, Internet of Things, NodeMCU, Bluetooth 5, Firebase, Android.

\section{INTRODUCTION}

In the same way that the notion of friend has been changing with the growth of mobile communications and social networks, where you can keep in touch even if you are not close to the person, the notion of home has also been changing with the growth of technology [1]. People take a little bit of home with them when they go out on the street, or go to work, and with that comes the concept of "digital houses". Digital houses adopt the use of digital equipment, such as computers and sensors, with the ability to communicate and interact with other equipment.
All of these concepts are strongly linked to the Internet of Things - IoT. This term was created by Kevin Ashton and it conveys the idea of connectivity, not only of computers on a network, but also of the most diverse types of things, such as monitoring, transport and household devices.

The International Data Corporation (IDC) expects global IoT spending to grow at a compound annual rate of $11.3 \%$ over the 2020-2024 period [2]. Furthermore, the Bluetooth Special Interest Group (SIG) expects a total of 1.81 billion annual Bluetooth smart home device shipments by 2024 , doubling the annual shipments of 2019 [3].

The various types of applications in home automation systems are commonly controlled by applications installed on devices such as smartphones or tablets. In addition, many different types of wireless connection technologies are also used, including ZigBee, Z-Wave, Bluetooth 4.0+ and Wi-Fi [4].

This work tries to take advantage of last progress of Bluetooth technology and cloud services to implement a home control and remote monitoring system.

This paper is organized as follows. The methodology used in the project is presented in this section, introducing the main idea of the project, presenting the technologies used through the work. Section 2 presents the system development describing the whole process for the electronic circuits constructed, while section 3 describes the results achieved. Finally, section 4 brings the conclusions highlighting the main test results obtained.

\section{Methodology}

This paper aims to describe the development of a home automation system composed of a central controller module, or hub, and some peripheral modules that interact with the environment, in the case of this project, a smart power plug and a LPG (Liquid Petroleum Gas) sensor. The system is controlled by an Android application. The Bluetooth 5.0 technology was used for the communication between the hub and peripherals, using the CC2640R2F microcontroller. 


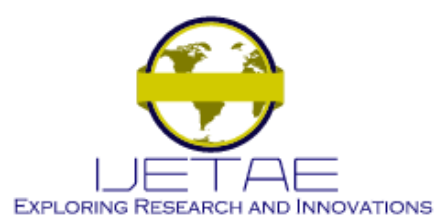

International Journal of Emerging Technology and Advanced Engineering

Website: www.ijetae.com (ISSN 2250-2459, ISO 9001:2008 Certified Journal, Volume 10, Issue 08, August 2020)

The data obtained from the peripherals are stored in a database in the cloud using the Firebase ${ }^{\circledR}$ platform, which can also be accessed by the Android $^{\circledR}$ application. The integration of the modules with the database was done using the NodeMCU ${ }^{\circledR}$ platform.

This section presents the proposed methodology to the system implementation. First, was defined the system architecture. Following, it was developed an App implemented in Firebase platform with the objective of store the data obtained from the sensors and actuators, data that can be accessed to take proper decisions. Next step consists in the implementation of the communication between NodeMCU and firebase platform. It enables establish the bidirectional data flow as NodeMCU can send and receive data form the data bank. The next step is the implementation of Bluetooth communications between the peripheral modules with the hub module. Also, it is written the firmware to allow communication between the Bluetooth modules and NodeMCU. With these stages completed it was written the code for the Android App using Android Studio IDE, that will act as the manmachine interface (IHM) of the system, and will allow the connection with the Firebase App. Then, it was implemented the hub and peripheral electronics. After that, it was completed the system integration.

In next section it is presented a brief review of different technologies that are used in this project.

\section{RELATED WORK}

In this section it is presented a brief review of technologies used in this project. Specifically, it is described main aspects of Bluetooth 5.0, Firebase and Android platform. The objective is to make clear how the control system proposed here can take advantage of these technologies to implement the home automation functionality and highlighting how these technologies are used in connection with this project.

\section{A. Bluetooth}

Bluetooth is a low-energy, low-cost, short-range wireless communication technology that enables the connection between devices in a small area, up to 10 meters, in most applications. It uses frequencies between 2.4 to $2.485 \mathrm{GHz}$, which belongs to industrial, scientific and medical applications [1].

In 1999, the Bluetooth SIG released the Bluetooth v1.0 specification and in 2002, the IEEE 802.15 committee approved the 802.15.1-2002 standard of the Wireless Personal Area Network, based on the Bluetooth SIG specification [5].
The Bluetooth versions 2.0 and 3.0, released in 2004 and 2009 , respectively, provided a higher rate of data exchange in communication. These settings became known as Bluetooth Basic Rate, or Bluetooth BR.

The Bluetooth 4.0 version, also known as Bluetooth Low Energy, Bluetooth LE, or just BLE, released in 2010, was the one that had the most impact in the IoT area. This version was developed in order to conserve energy and work with sensors or other low consumption devices, in addition to being retro compatible with previous versions.

Figure 1 shows the architecture of the Bluetooth Low Energy protocol stack. The GATT layer, an acronym for Generic Attribute Profile, handles the data to be transmitted between devices. The GAP layer, acronym for Generic Access Profile, is responsible for defining how one device will discover the other and how connections will be created or broken [6]. The HCI layer carries commands and events between the Host and Controller areas. The Link layer defines the state of the device's radio, depending on the state defined by the GAP. The physical layer, or PHY, is the lowest layer of the stack. It configures the physical parameters of data transmission and reception, such as signal strength and range [7].

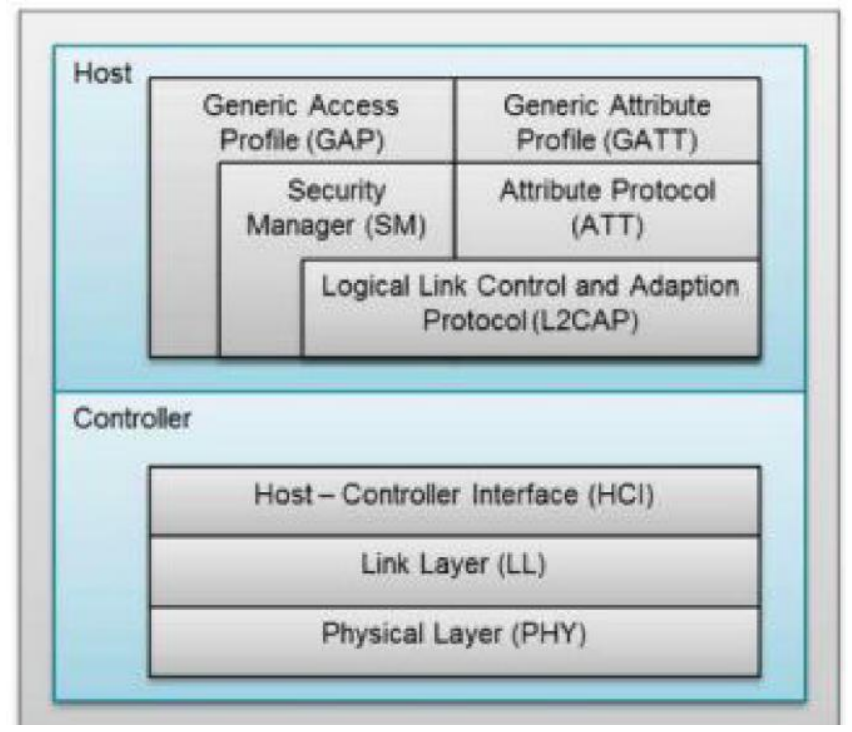

Figure 1. Bluetooth LE protocol stack [7]

Bluetooth 5.0 version, released in 2016, was developed implementing improvements and new concepts aiming at total integration with the IoT scenario. The main changes were to increase the data rate to the double of the fourth version, increase the data range to up to four times the range of the fourth version and increase the advertising package [8]. 


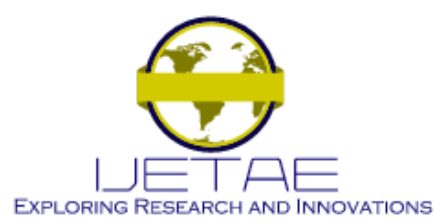

International Journal of Emerging Technology and Advanced Engineering

Website: www.ijetae.com (ISSN 2250-2459, ISO 9001:2008 Certified Journal, Volume 10, Issue 08, August 2020)

For the Bluetooth application of this project, the Texas Instruments CC2640R2F microcontroller was chosen. This microcontroller is part of the SimpleLink Ultra-Low Power line, low cost, and has support for Bluetooth 4.2 and Bluetooth 5.0. For familiarization with the microcontroller, the LAUNCHXL-CC2640R2 development board was chosen. This board has a CC2640R2FRGZT microcontroller, an on-board antenna, and connectors with access to all GPIOs (General Purpose Input/Output). The board also has a TM4C1294NCPDT microcontroller, from Texas Instruments, which emulates a JTAG debugger, for debugging and load the programs into the CC2640R2F, in addition to providing a USB-Serial interface for communication with the CC2640R2F through UART. For the validation of the communication between the microcontrollers, and for the implementation of the project's peripheral modules, two standalone boards were made based on the development kit, as can be seen in figure 2. The boards have an on-board antenna and connectors that give access to the GPIOs. To load the firmware into the microcontroller, the LaunchKit was used.

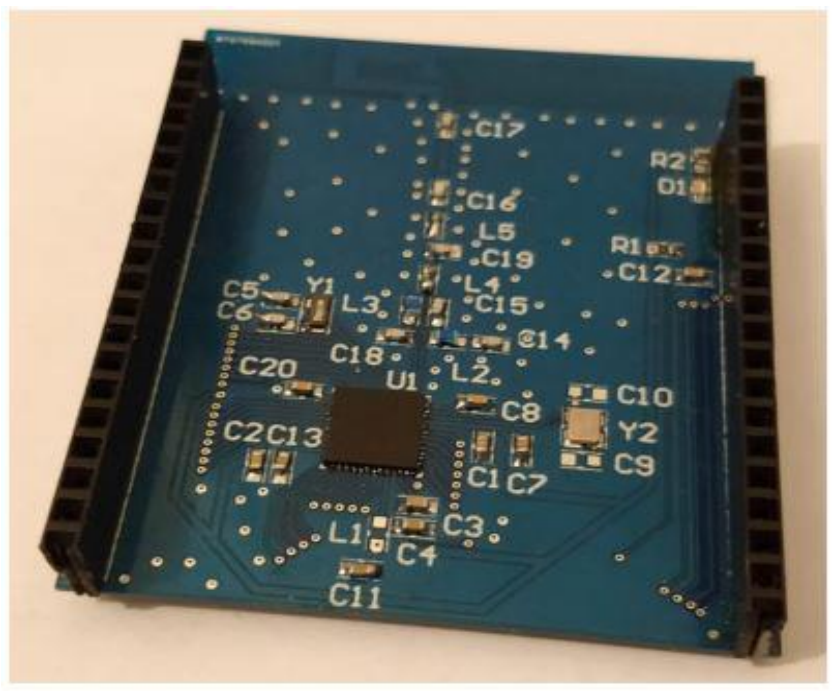

Figure 2. Standalone board built based on the CC2640R2F microcontroller

\section{B. Wi-Fi}

The protocol used by the Wi-Fi Wireless Local Area Network (WLAN) is defined by the IEEE 802.11 specification. The IEEE 802.11 standards were first published in 1997, and have changed over the years several times [5]. The stack used by Wi-Fi follows the TCP/IP (Transmission Control Protocol/Internet Protocol) standards, as shown in figure 3 .
The lowest layer is composed of the physical layer, MAC layer and Logical Link Layer. The topmost layer deals with the Internet, the transport of information and the application [5].

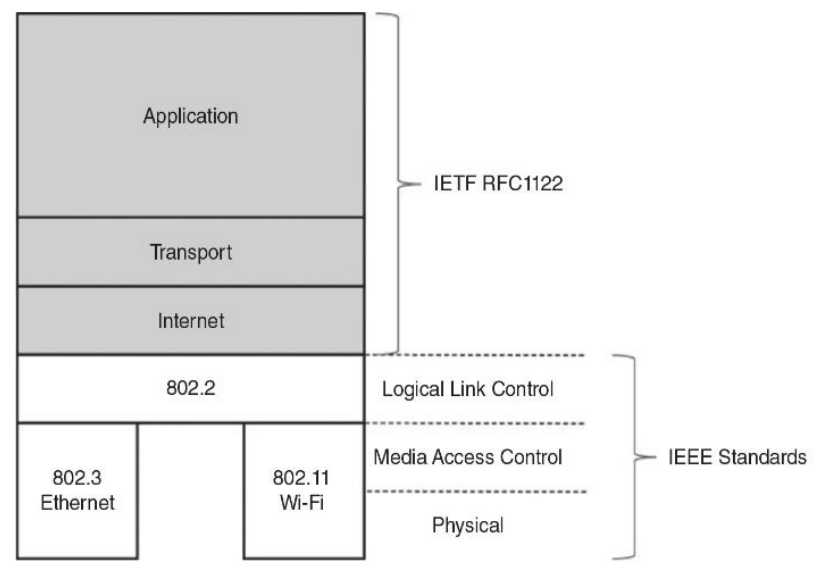

Figure 3. Wi-Fi protocol stack [5]

For the internet applications of this project, the NodeMCU DevKit V1.0 module was chosen as the Wi-Fi interface for the hub. The NodeMCU DevKit is a development platform for IoT, open source, and is used together with the open source firmware NodeMCU. It is based on ESP8266 from Espressif Systems and was originally developed to run applications made with the Lua programming language, but there is the possibility of using the Arduino platform to programming purposes which will be the case for this project.

\section{Firebase}

Firebase is a BaaS (Backend-as-a-Service) platform from Google that provides several tools for the development of mobile and WEB applications. BaaS platforms are services that allow an application to connect to cloud services, making it possible to implement a database remotely and send notifications [9]. Firebase Realtime Database is a NoSQL database hosted in the cloud. The data is stored as JSON (JavaScript Object Notation) and synchronized in real time with all connected clients [10]. In this project, the Firebase Realtime Database was used to store all the peripherals data, allowing for the Android application to acquire these data or update them.

\section{Android Platform}

Android is an open source operating system based on the Linux platform, commonly used on mobile devices such as smartphones [11]. Google acquired the company Android Inc in 2005. 


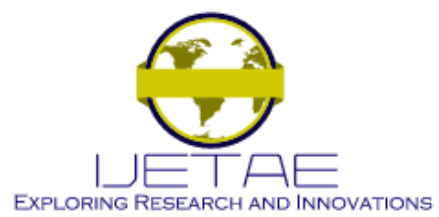

International Journal of Emerging Technology and Advanced Engineering

Website: www.ijetae.com (ISSN 2250-2459, ISO 9001:2008 Certified Journal, Volume 10, Issue 08, August 2020)

In 2007 the Open Handset Alliance (OHA) was created, a group of companies led by Google to develop products related to mobile devices [11].

Android applications are made using the programming languages XML and Java. XML is responsible for the graphical interface and application settings, while Java is responsible for functionality [11]. In this project, an Android application was used for the communication with the Firebase server by a smartphone, thus controlling the peripherals.

\section{SYSTEM DESIGN}

The entire development was based on the block diagram shown in figure 4. The Smart Power Plug peripheral consists mainly of a standalone module containing the CC2640R2F microcontroller, which controls a relay module, allowing or not the connection to the electrical network of the device connected to the peripheral. The LPG Sensor peripheral also consists of a standalone module containing its own CC2640R2F microcontroller. However, it receives information from a MQ-5 LPG sensor, indicating whether or not gas is present. Both peripherals communicate directly with the central hub, which consists of a CC2640R2F LaunchKit and a NodeMCU DevKit. Therefore, each peripheral has its own CC2640R2F microcontroller, which communicates via Bluetooth with the CC2640R2F microcontroller present in the central hub, which is a bilateral communication, as shown in figure 4 . The central hub receives and sends the information to a Firebase Realtime Database. This information contained in the database can be consulted through a smartphone with the Android application developed in this project.

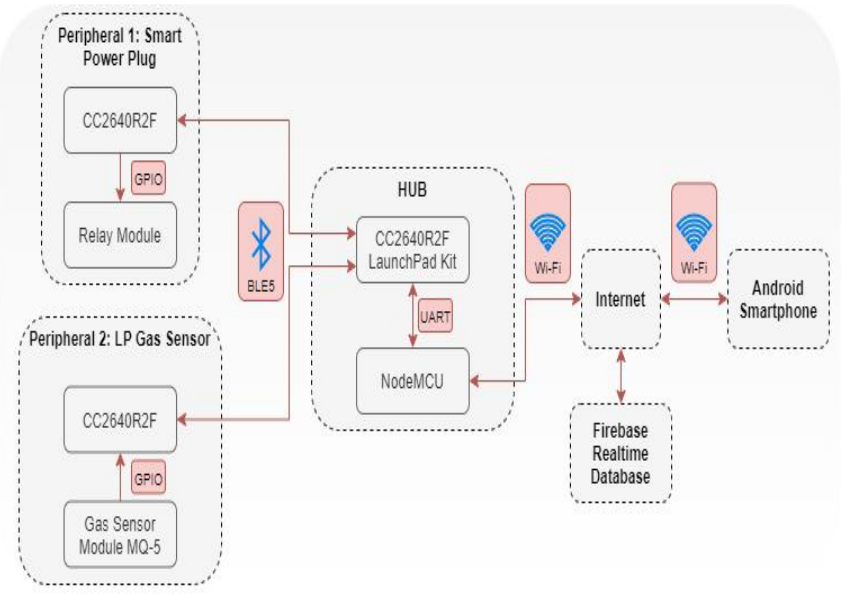

Figure 4. System block diagram

\section{A. Hardware implementation}

For practical reasons, in order to be able to use the CC2640R2F microcontroller in the design of the peripherals, standalone printed circuit boards were made, since the microcontroller itself is small in size and the access to the pins would be of great difficulty. The main idea was to create boards that had the minimum resources as necessary for adequate operation of the microcontroller. Also, it allows easier access to microcontroller's pins and allows each peripheral to have its own microcontroller, leaving the CC2640R2F LaunchKit to be used only on the central hub. The top and bottom of the final board layout is shown in figure 5.
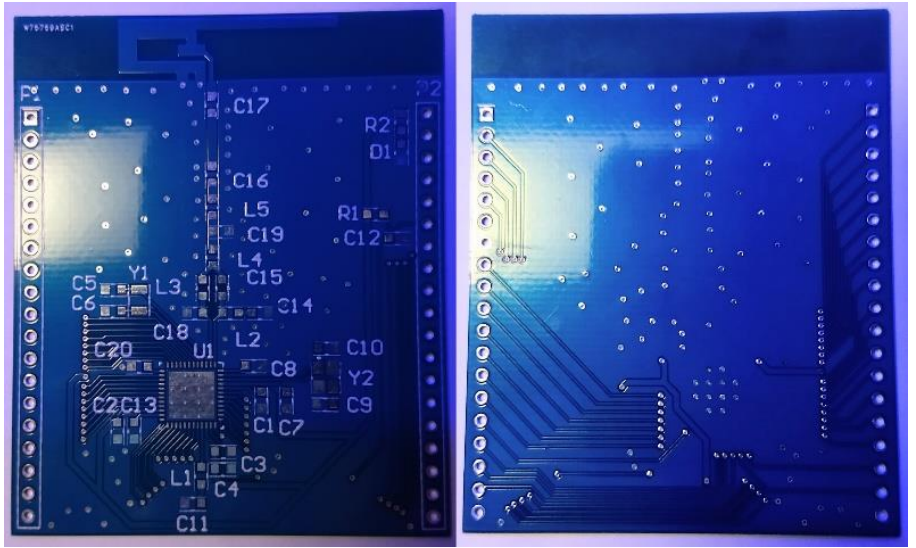

Figure 5. Printed circuit board produced for the project

\section{B. Smart Power Plug Peripheral Design}

The central elements of the Smart Power Plug peripheral are the standalone board, containing the CC2640R2F microcontroller, which communicates via Bluetooth with the central hub, the relay module, which acts as an electromechanical switch, and a push button, which activates the relay. The block diagram in figure 6 shows the main components used in the elaboration of the Smart Power Plug peripheral.

Due to the device being connected directly to an outlet, with a voltage of 127 or 220 volts, a Hi-Link HLK-PM01 power supply was used to power the peripheral components. This source was chosen because it supports voltages from 100 to 240 volts at its input, has a regulated output of 5 volts, supporting 3 watts of power, in addition to being compact in size.

Two LEDs were also added to the circuit. The first one, a green LED, indicates that the circuit is on. The second one, a red LED, indicates whether the relay is activated or not. 


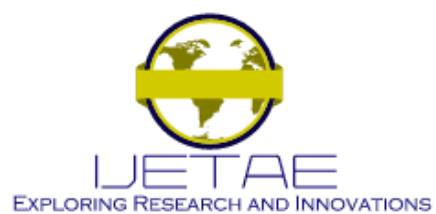

International Journal of Emerging Technology and Advanced Engineering

Website: www.ijetae.com (ISSN 2250-2459, ISO 9001:2008 Certified Journal, Volume 10, Issue 08, August 2020)

To connect the device to the outlet, a conventional outlet adapter was used. The final assembly of the device is shown in figure 7.

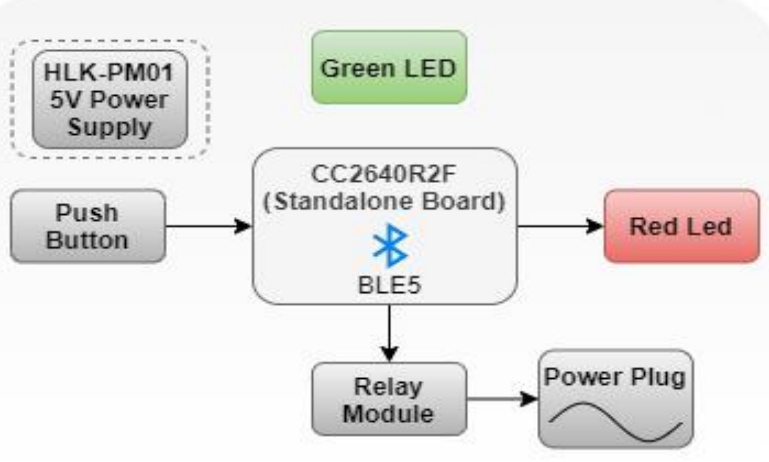

Figure 6. Block diagram of the main components contained in the peripheral Smart Power Plug

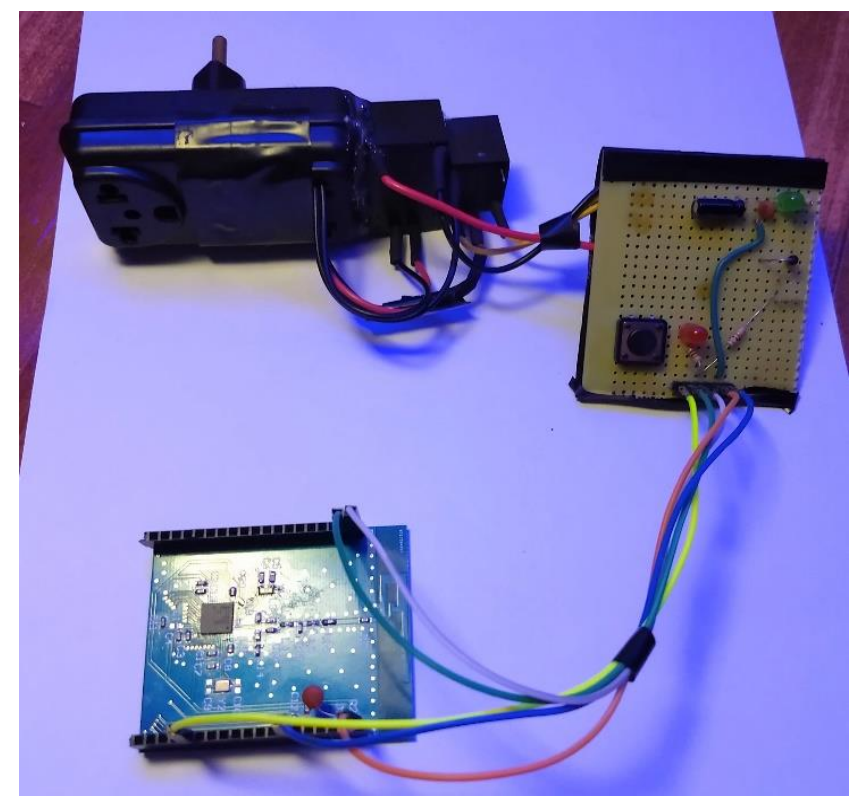

Figure 7. Final assembly of the Smart Power Plug peripheral

\section{LPG Sensor Peripheral Design}

For the development of the LPG Sensor peripheral, a standalone board containing the CC2640R2F microcontroller was also used, which communicates via Bluetooth with the central hub, in addition to a LPG module and a battery to energize the system. The main components used are shown in figure 8.
For the gas sensing, the sensor module MQ-5 was used. This module operates with 5 volts at its input, has a sensor with high sensitivity for LPG, has a digital and an analog output, to inform the presence of the gas, and an adjustable potentiometer to regulate its sensitivity.

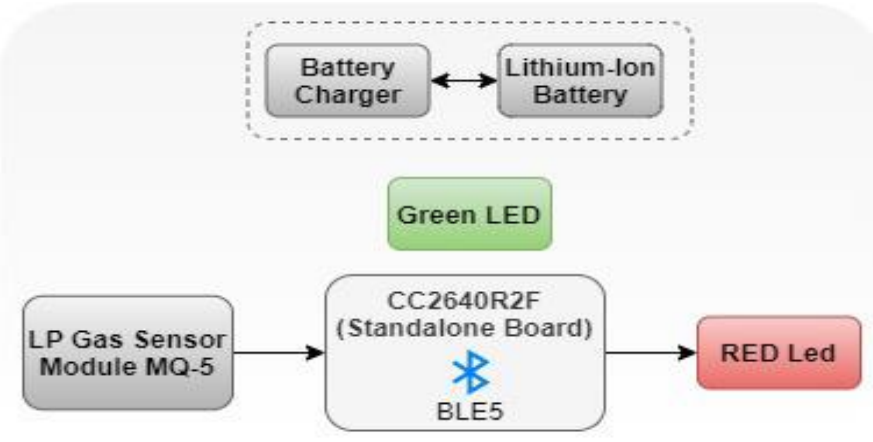

Figure 8. Block diagram of 1he main components contained in the LPG Sensor peripheral

As this peripheral can be installed in different locations, it was decided to power it using a 3.7-volt nominal lithiumion battery, to avoid the need for an outlet close to the device. Two LEDs were also included, one green LED, to indicate when the device is on, and one red, to indicate the presence or absence of gas. The complete assembly of the LPG Sensor peripheral is shown in figure 9.

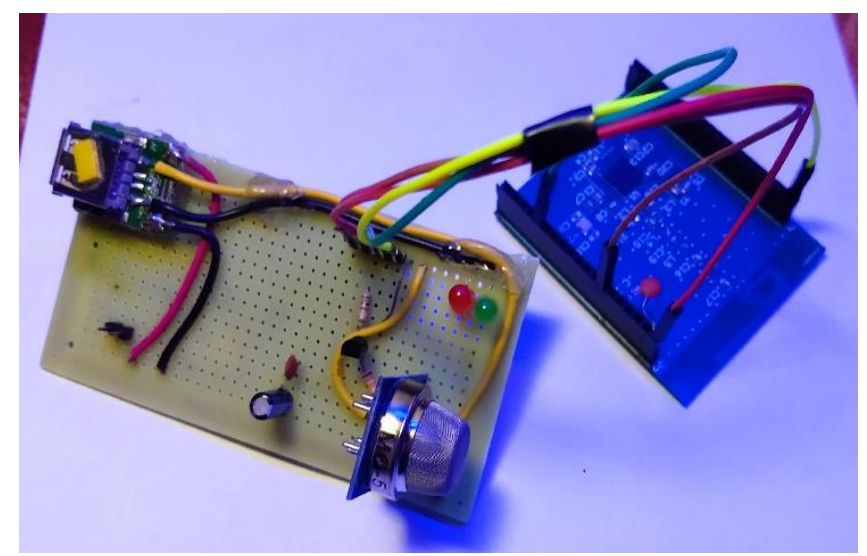

Figure 9. Complete assembly of the LPG Sensor peripheral

\section{Central Hub Design}

The central hub, unlike the peripherals, was mounted on a protoboard not requiring a separate standalone board, and the CC2640R2F of this LaunchKit was used as the basis for Bluetooth communication. 


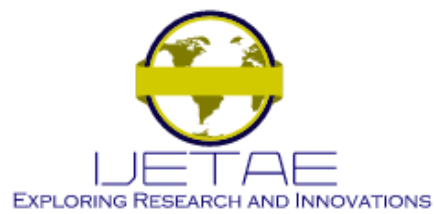

International Journal of Emerging Technology and Advanced Engineering

Website: www.ijetae.com (ISSN 2250-2459, ISO 9001:2008 Certified Journal, Volume 10, Issue 08, August 2020)

For the Wi-Fi communication, the NodeMCU DevKit module was used. Figure 10 shows the block diagram containing the main components used in the development of the central hub.

Three indication LEDs were included in the assembly: A green LED to indicate the presence of energy, a yellow LED to provide information on the status of the $\mathrm{Wi}-\mathrm{Fi}$ connection, and a red LED to provide information on the status of the Bluetooth connection. In addition to the LEDs, two push buttons were included, the first one to reset the Wi-Fi settings, and the second to initiate a Bluetooth connection.

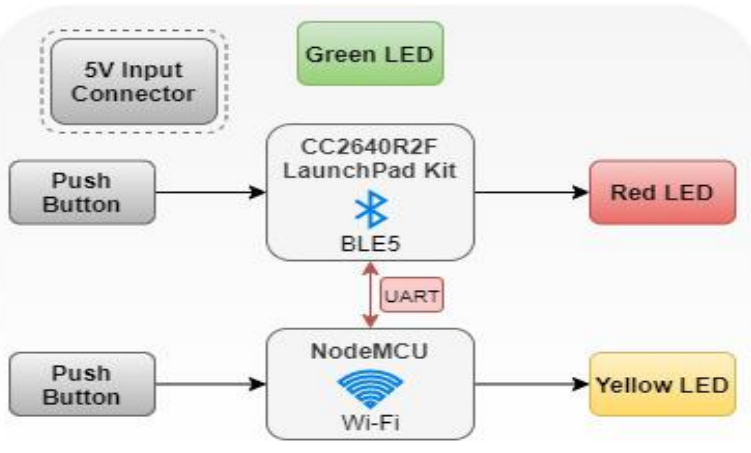

Figure 10. Block diagram of the main components contained in the central hub

The final assembly of the hub is shown in figure 11.

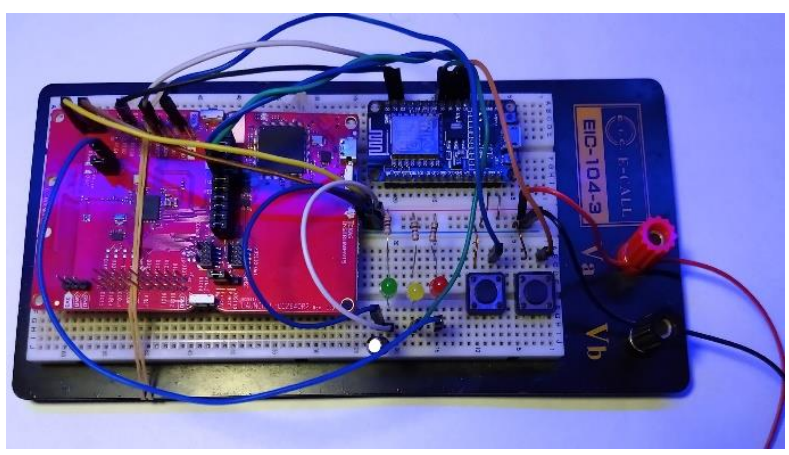

Figure 11. Complete assembly of the central hub

\section{E. Firmware}

As a basis for the development of the firmware, the microcontroller manufacturer, Texas Instruments, provides a Software Development Kit, or SDK, called "SimpleLink CC2640R2 SDK", containing documentations, examples and base codes for the microcontroller [12]. The code implements a Real Time Operating System, or RTOS, developed by Texas Instruments and called TIRTOS.
RTOS is a more robust way of managing tasks within an application, minimizing delays [12]. The code has two projects within it. The first one, called Stack Library, implements the Bluetooth Host and Controller protocols, where communication is actually carried out. The second, called "App", manages the application and communicates directly with the Stack Library. In this project, only the "App"project was modified, to meet the requirements of the desired application. The TI-RTOS operates by managing tasks, and these tasks are created or communicated from messages that are scheduled in a stack, and later processed by a state machine at the appropriate time.

\section{F. Smart Power Plug Peripheral Firmware}

For the Smart Power Plug peripheral, the firmware for the CC2640R2F microcontroller was written. For the development of the firmware, the base code entitled "ble5_project_zero", from version 3.30 of the SDK, was used. This code already has some structures, functions and state machines previously implemented, which can be modified according to the needs of each application. The main functions previously implemented used during the development are presented in the table 1 .

TABLE I

MAIN FUNCTIONS PREVIOUSLY IMPLEMENTED USED DURING THE DEVELOPMENT OF THE PERIPHERALS FIRMWARE.

\begin{tabular}{|l|l|}
\hline Function & Description \\
\hline init() & $\begin{array}{l}\text { Called during initialization, } \\
\text { containing all the parameters for the } \\
\text { system to initialize. }\end{array}$ \\
\hline $\begin{array}{l}\text { processApplicationMessa- } \\
\text { ge() }\end{array}$ & $\begin{array}{l}\text { State machine that process the } \\
\text { messages sent by the application. }\end{array}$ \\
\hline processGapMessage() & $\begin{array}{l}\text { State machine that process the } \\
\text { messages related to the GAP coming } \\
\text { from the application and from the } \\
\text { BLE stack. }\end{array}$ \\
\hline
\end{tabular}

Firstly, for the firmware implementation, the service that the Bluetooth application will execute was created, defining its Universally Unique Identifier, or UUID of the service, the UUID of the characteristic, the size of the characteristic and its properties, as write, read and notify. To create the service structure and the files to be included in the firmware, the Service Generator, available at SimpleLink Academy 3.30, from Texas Instruments, was used. 


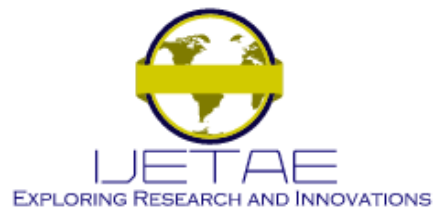

International Journal of Emerging Technology and Advanced Engineering

Website: www.ijetae.com (ISSN 2250-2459, ISO 9001:2008 Certified Journal, Volume 10, Issue 08, August 2020)

After including the files containing the service definitions in the project, the name of the device and the advertisement message were modified in the file "project_zero.c" to include the word "power plug". The advertisement will be used for the hub to recognize the type of device to which it should connect. The microcontroller pins that control the LED and the relay, as well as the characteristic $0 \mathrm{xABC} 1$, have their states changed, when the push button is pressed, calling the function "buttonDebounceSwiFxn()", and from the central hub via Bluetooth, calling the function "user_tomada_service_ValueChangeCB()". The flowchart in figure 12 shows the operation of the firmware.

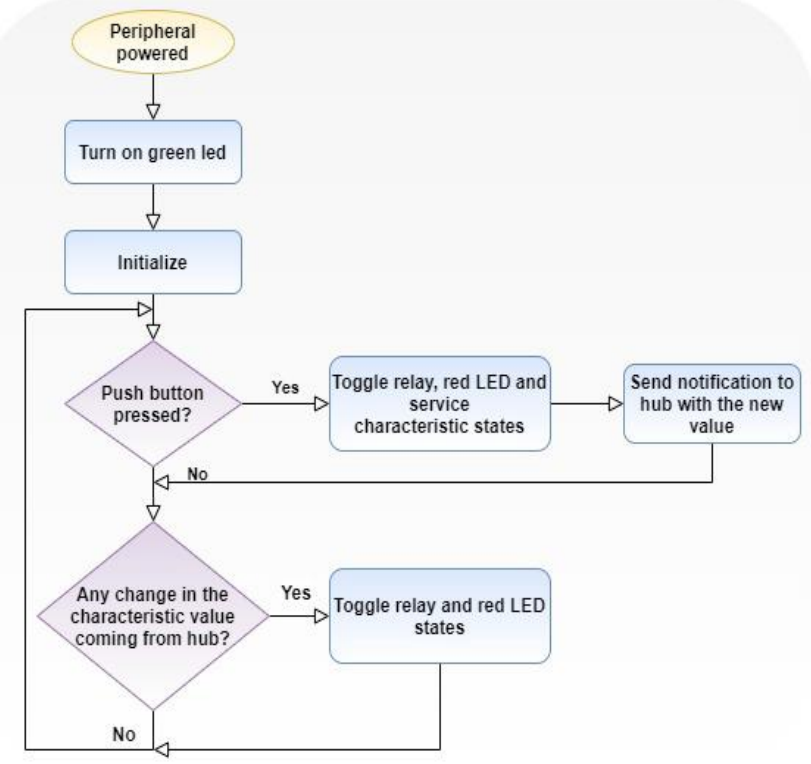

Figure 12. Flowchart of the operation of the firmware developed for the Smart Power Plug peripheral

Depending on the signal strength, the device automatically updates the PHY, using a state machine contained in the "updatePHYStat()" function. When the devices are closer and the signal strength is strong, preference is given to the PHYs with the highest communication speed, such as $2 \mathrm{Mbps}$ and $1 \mathrm{Mbps}$. When the signal strength is low, preference is given to PHY CODED.

\section{G. LPG Sensor Peripheral Firmware}

The process of implementing the LPG Sensor peripheral firmware was similar to that of the Smart Power Plug and the same main functions were used. Firstly, the service to be processed was defined and created using the Texas Instruments Service Generator.
As this peripheral does not receive any command from the central hub, it has not been assigned the write property.

As the hub can identify the peripheral, the name "gas sensor" was assigned to it, adding the same name to the advertisement message. When the pin of the microcontroller that is connected to the sensor undergoes a state transition caused by the presence or absence of gas, the "buttonCallbackFxn()" function is called, starting a 200 millisecond clock. After the end of the clock, the function "onOffDebounceSwiFxn()" is called, which reads the logic level of the pin and schedules the "PZ_ON_OFF_EVT" process. The "PZ_ON_OFF_EVT" process assigns the logic level value to characteristic $0 \times \mathrm{ABC} 1$ and the LED pin, turning it on or off. The flowchart of the firmware operation, is shown in figure 13.

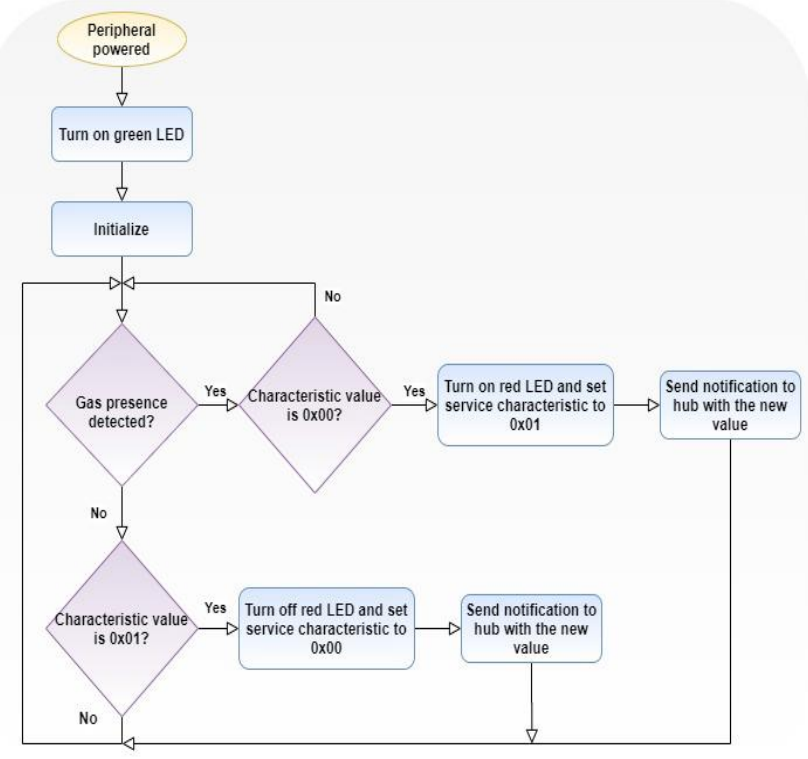

Figure 13. Flowchart of the operation of the Firmware developed for the LPG sensor peripheral

\section{H. Central Hub Firmware}

For the development of the CC2640R2F microcontroller firmware, contained in the central hub, the base code entitled "ble5_simple_central", from version 3.30 of the SimpleLink CC $2640 \mathrm{R} 2$ SDK, was used. Just as in the case of the "ble5_project_zero"code, used in the development of the peripherals firmware, the "ble5_simple_central" has several structures previously implemented, functions and state machines, which can be modified according to the application's needs. The main functions previously implemented used during the development are presented in the table 2 . 


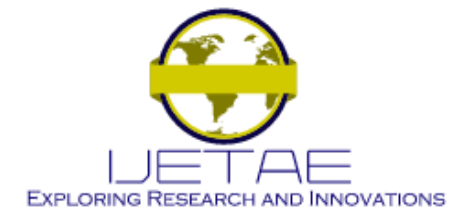

International Journal of Emerging Technology and Advanced Engineering

Website: www.ijetae.com (ISSN 2250-2459, ISO 9001:2008 Certified Journal, Volume 10, Issue 08, August 2020)

TABLE 2

MAIN FUNCTIONS PREVIOUSLY IMPLEMENTED USED DURING THE DEVELOPMENT OF THE CENTRAL HUB FIRMWARE

\begin{tabular}{|l|l|}
\hline Function & Description \\
\hline init() & $\begin{array}{l}\text { Called during the initialization, containing } \\
\text { all the parameters for the system to } \\
\text { initialize }\end{array}$ \\
\hline processAppMsg() & $\begin{array}{l}\text { State machine that process the messages } \\
\text { sent by the application }\end{array}$ \\
\hline processGATTMsg() & $\begin{array}{l}\text { State machine that process the messages } \\
\text { related to the GATT coming from the } \\
\text { application and from the BLE stack. }\end{array}$ \\
\hline processGapMsg() & $\begin{array}{l}\text { State machine that process the messages } \\
\text { related to the GAP coming from the } \\
\text { application and from the BLE stack. }\end{array}$ \\
\hline processGATTDiscEvent() & $\begin{array}{l}\text { State machine that process the messages } \\
\text { related to the Discovery process of the } \\
\text { GATT }\end{array}$ \\
\hline
\end{tabular}

When the value of the peripheral characteristic is changed, it sends a notification via Bluetooth to the hub, which sends this value to NodeMCU via UART. In the case of the Smart Power Plug, this process occurs when the button is pressed. For the Gas Sensor, this process occurs when the sensor detects the presence of gas or not. When NodeMCU sends a change of value to the hub, via UART, the hub writes this new value in the peripheral characteristic. Figure 14 shows the flowchart of the operation of the developed firmware.

When a peripheral is disconnected or the connection is lost, the "GAP_LINK_TERMINATED_EVENT" process is scheduled, which when executed by the "processGapMsg()"function, deletes the device from the list and, if there are no more devices connected, turns off the red LED. For the integration of the data used by peripherals with the cloud, a real-time database from Firebase was created. The data structure defined to be used in the database is shown in figure 15. The "Devices" substructure stores all peripherals of a given user, including their identification key, their type and their value. The "Users" sub-structure stores the users' information, including their email, full name, password and username.

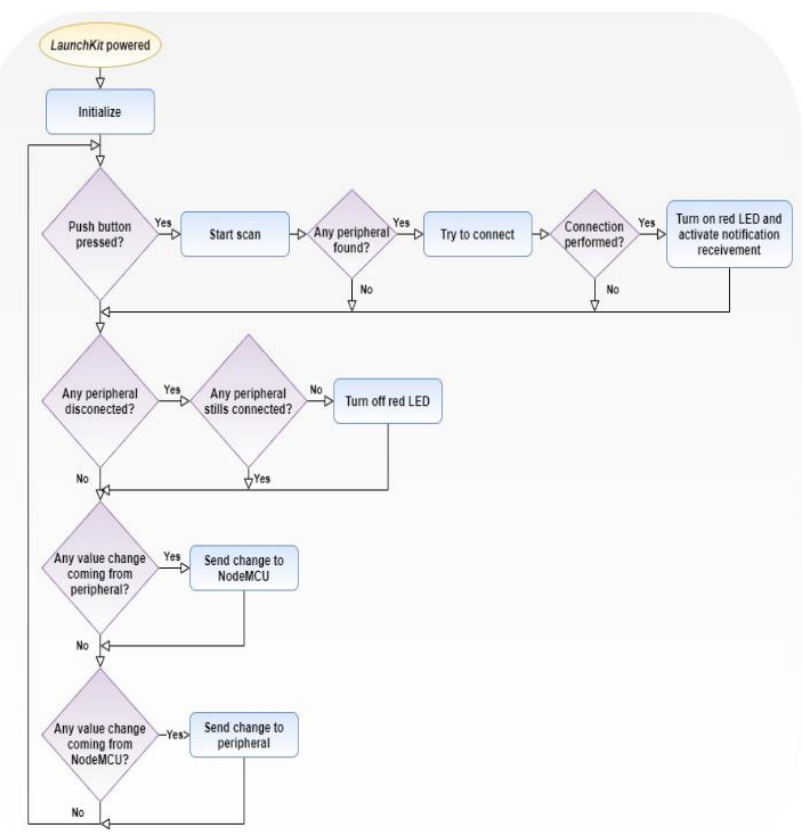

Figure 14. Flowchart of the operation of the firmware developed for the CC2640R2F LaunchKit used in the hub

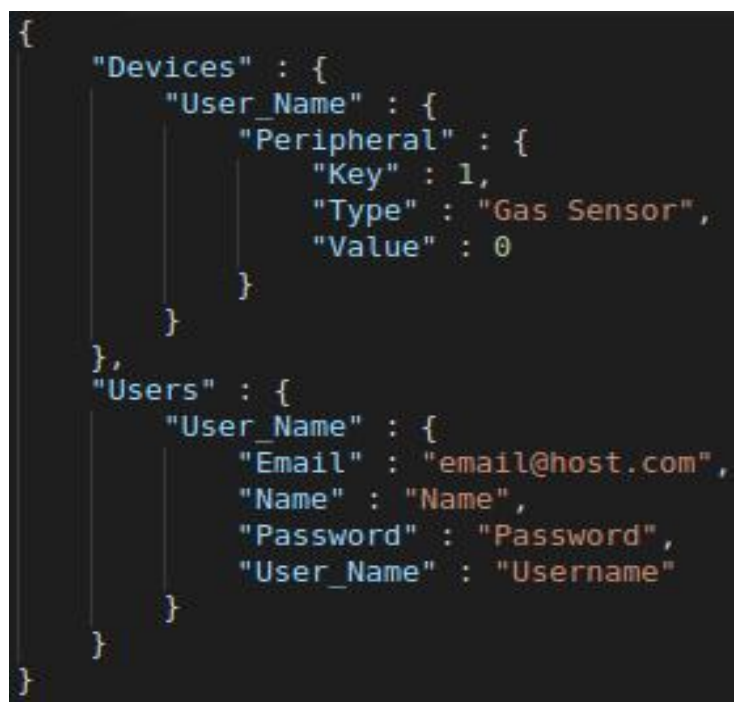

Figure 15. Data structure used in the Firebase database

For the communication with the CC2640R2F LaunchKit and the Firebase database, the firmware for the NodeMCU was written. The flowchart in figure 16 shows the operation of the developed firmware. 


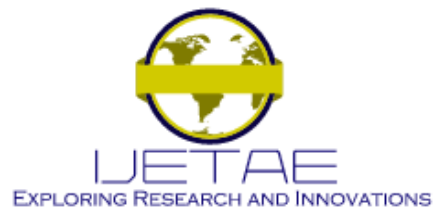

International Journal of Emerging Technology and Advanced Engineering Website: www.ijetae.com (ISSN 2250-2459, ISO 9001:2008 Certified Journal, Volume 10, Issue 08, August 2020)

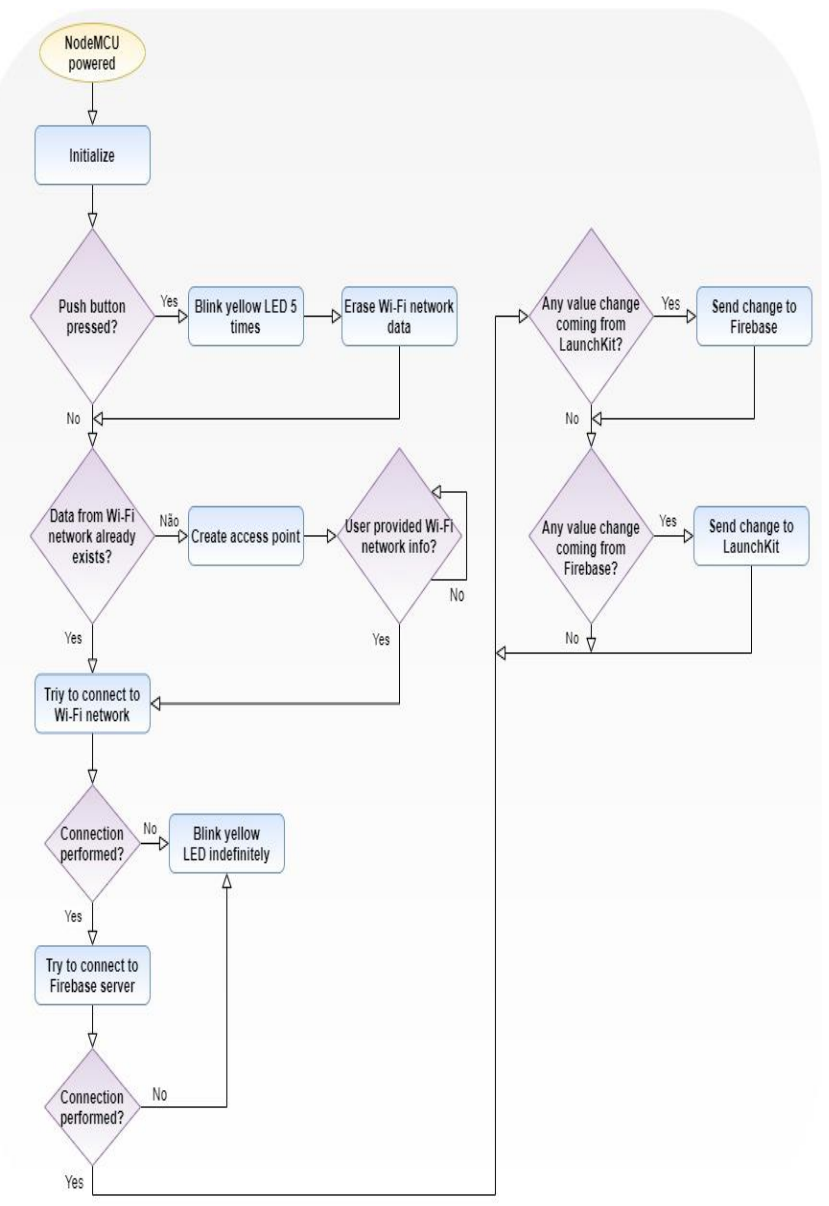

Figure 16. Flowchart of the firmware developed for the NodeMCU used in the hub

In the "setup()"function, it is checked if there are already data regarding the Wi-Fi network and the user's registration in the non-volatile memory. If it does not exist, a Wi-Fi access point is generated where the user can register his information. If data already exists, the program connects to theWi-Fi network automatically. In order to the NodeMCU be able to reset the data of theWi-Fi network, at the beginning of the code, the state of the pin that is connected to the reset button is checked. If the button is pressed, the yellow LED will flash five times and the data will be reset.

Then, the connection to the Firebase server is initiated, using the Uniform Resource Locator, or URL, of the Firebase database and its authentication key, both available in the server settings on the Firebase website. Then, the code compares the registration password entered by the user with the password stored in the database.
If they do not match, the yellow LED remains blinking indefinitely. If the passwords match, the program enters the stream mode using the "stream()"method of the Firebase class, checking if there has been a change in the values of the user's devices. After starting the communication with the Firebase server, the yellow LED is turned on. In the "loop()"function, when the presence of a new message from the CC2640R2F LaunchKit is noted, the program checks whether the message is valid, and if so, updates the database with the new value assigned to the peripheral indicated in the message. If values are updated from the Firebase database, it is checked which device the message comes from, and a change of value message is sent via UART to the CC2640R2F LaunchKit.

The Android application was developed using the Android Studio software and was divided into four different screens. The code for the implementation of the first screen, called main screen, was made using the flowchart of figure 17 as basis. Firstly, the modules "com.google.firebase:firebase-auth:18.0.0" and "com.google.firebase: firebase-database:18.0.0" were added to the "build.gradle" file at the application level, so that it was possible to implement communication with the Firebase server. Then, the layout of the main screen was created, adding two text boxes, one for filling in the email and one for the password. Two buttons were also created, one to login and the other to register.

Then, a class called "User" was implemented, which stores the user name, full name, email and password of the user. The main activity was also created, which implements the activity of the main screen. When created, the activity checks whether the application instance is already associated with a Firebase account. If so, the devices view screen opens automatically. If there is no account associated, and the email and password fields are filled in with an existing account, when pressing the "ENTER" button, the login is made. If the button "REGISTER" is pressed, the registration screen opens.

The code for the implementation of the application registration screen was made using the flowchart of figure 18 as the basis. The registration screen layout was made similarly to the main screen, creating the screen layout with text boxes to store the user name, full name, email and password, in addition to two buttons, one to confirm the registration and the other to cancel. After creating the screen layout, its activity was implemented.

When the screen is created, it is possible to fill in the username, full name, e-mail and password fields. 


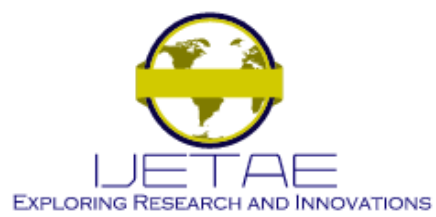

International Journal of Emerging Technology and Advanced Engineering Website: www.ijetae.com (ISSN 2250-2459, ISO 9001:2008 Certified Journal, Volume 10, Issue 08, August 2020)

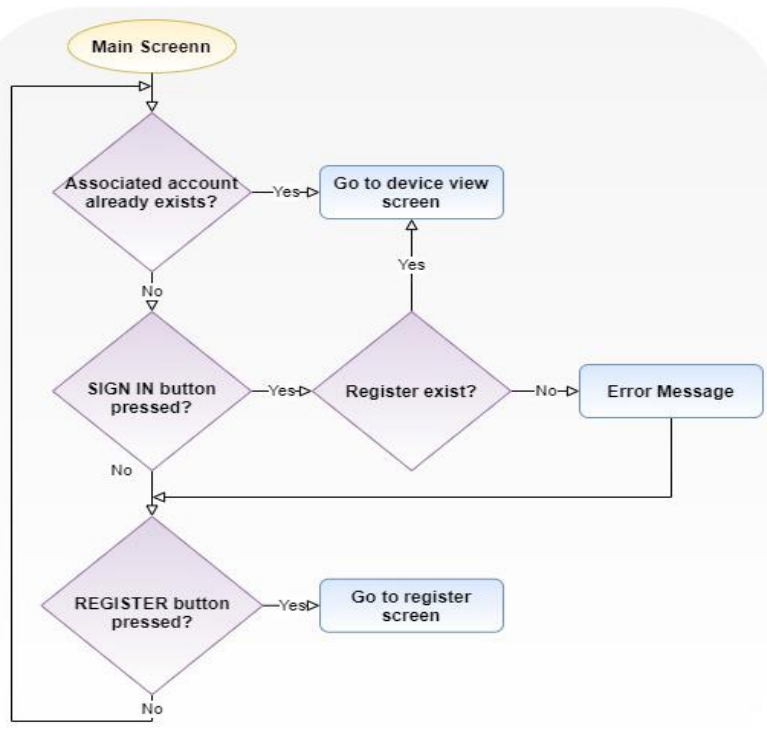

Figure 17. Flowchart of the operation of the Main Screen developed for the Android application

When the "REGISTER"button is pressed, the field information is retrieved, and a new user is created in the Firebase database. After the registration has been executed, the application is redirected to the main screen so that the login can be made. The screen also has a cancel button, which returns to the main screen without performing any other process.

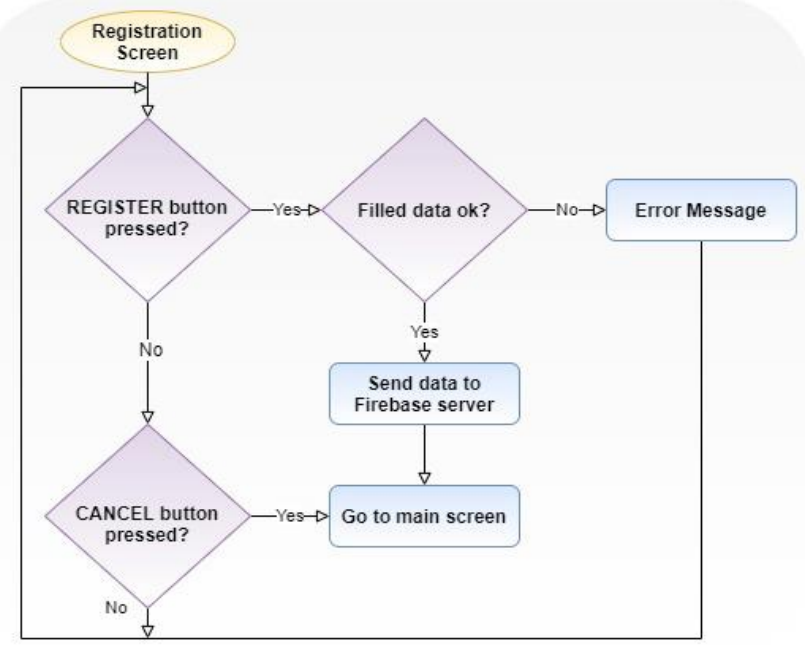

Figure 18. Flowchart of the operation of the Registration Screen developed for the Android application

The code for implementing the device view screen of the application was made using the flowchart of figure 19 as the basis.
To create the screen, first a class called "Device" was created that stores the device type, its identification key and the value of their state. Then, the screen layout was created, in the same way as for the other screens. Due to the user being able to add one or more devices, to implement the layout, the widget called "RecyclerView" was used, including the module "androidx.recyclerview: recyclerview:1.0.0" to the file "build.gradle", in application level. With this widget, it is possible to create and display on the screen a list of objects of variable size.

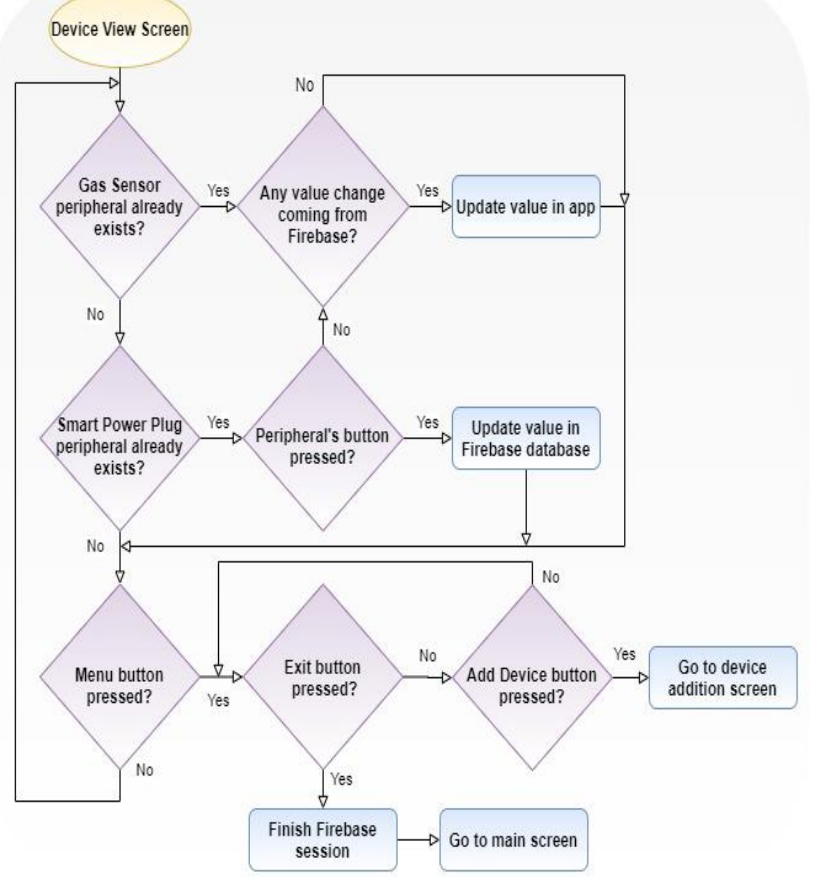

Figure 19. Flowchart of the Device View Screen developed for the Android application

After that, an adapter called "DeviceAdapter" was implemented, which inherits the "RecyclerView.adapter" class. With this adapter, it is possible to manage the objects that RecyclerView displays on the screen. Then, the activity of the devices view screen was implemented. This activity checks if the user has registered devices. If so, these devices are recovered and sent to the adapter, which displays device information on the screen. The devices values are monitored continuously. When an object on the screen referring to the Smart Power Plug device is pressed, the adapter switches the state of the device, sending a new value to the Firebase database, which is the opposite of what was previously there. 


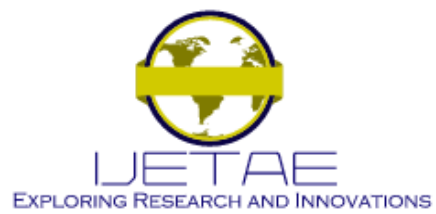

International Journal of Emerging Technology and Advanced Engineering

Website: www.ijetae.com (ISSN 2250-2459, ISO 9001:2008 Certified Journal, Volume 10, Issue 08, August 2020)

A menu-type resource has also been created. This feature includes an options menu on the top bar of the application, containing two options, namely "Add Devices" and "Log Out". When any of the menu options is pressed, the chosen option is checked. If the option is to add new devices, the device addition screen opens. If the option chosen is to log out, the session is closed with the Firebase server, and the main screen is opened.

The code for implementing the application's device addition screen was made using the flowchart in figure 20 as the basis. Firstly, the screen layout was implemented, containing two buttons, one to add the Smart Power Plug peripheral, and one to add the Gas Sensor peripheral.

Then, the screen activity was created. By clicking on the "Smart Power Plug" button, an object of the type "Smart Power Plug" is created in the Firebase database for the registered user. Within this object, a list is created with an object for the value, which stores the state of the peripheral, which can be "1"or "0", an object for the peripheral identification key, having the value "1" for the peripheral Smart Power Plug and the value "2" for the peripheral Gas Sensor, and an object for the type of device, which stores the value "Smart Power Plug" or "Gas Sensor". The exact same procedures were done for the button "Gas Sensor".

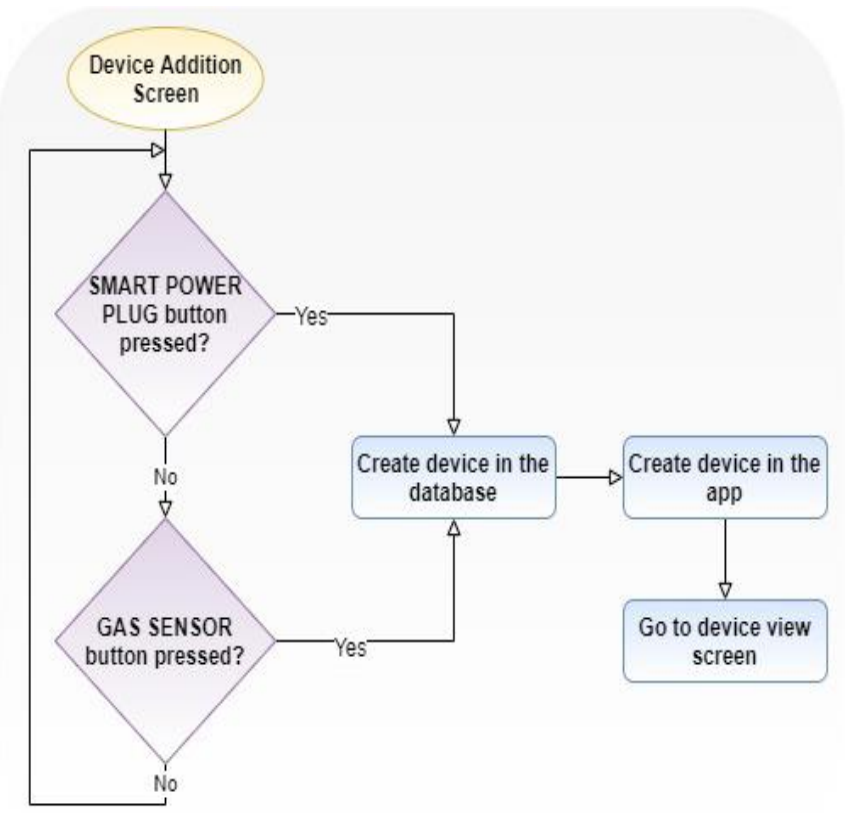

Figure 20. Flowchart of the operation of the Device Addition Screen developed for the Android application

\section{TEST RESUlts}

Practical main results are presented in this section.

\section{A. Peripheral test}

To be able to test the operation of both peripherals, the mobile application nRF Connect, version 4.24.1, was used. In the application, when performing the scanning process, when a new device is found, it is possible to view some information about it, such as its name, its address, the type of device, the strength of the receiving signal, among other information.

When connecting to the device, the application shows all the features of the service. As shown in figure 21 , on the left side, the characteristic of UUID $0 x A B C 1$ of the UUID 0xABC0 service of the Smart Power Plug device, is presented, and the service has the name "Unknown Service". Through the application, it is possible to read, write and receive notifications of the characteristic, and the icons of these options are arranged next to the service name. For the case of the Gas Sensor peripheral, as shown in figure 21 , on the right side, only the options to read and receive notification are enabled.

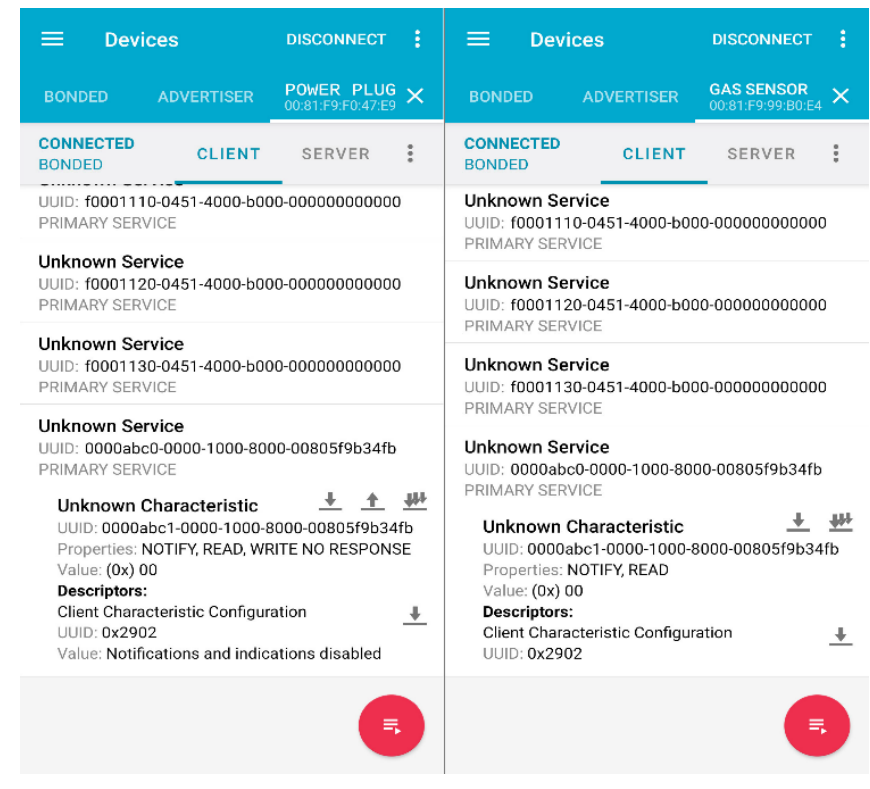

Figure 21. Scanning and connecting the nRFConnect application to the Smart Power Plug peripheral

Through the application, it was also possible to compare the peripheral antenna performance in relation to the LaunchKit antenna. 


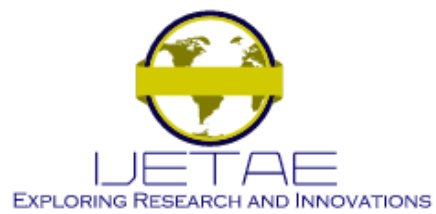

International Journal of Emerging Technology and Advanced Engineering

Website: www.ijetae.com (ISSN 2250-2459, ISO 9001:2008 Certified Journal, Volume 10, Issue 08, August 2020)

To do it, the code "ble5_project_zero"was loaded in LaunchKit, which was used as a basis for the development of the peripherals' firmware. With the devices arranged at approximately the same distance, and with the antennas in the same orientation, all the signals strength was close, around $-61 \mathrm{dBm}$, showing that the antenna layout and the coupling circuit were in accordance with the design recommendations.

These power values were used only to obtain an estimate, as they vary slightly over time, and are dependent on the type of antenna, the coupling circuit, the antenna arrangement, the objects around the antenna, external electromagnetic interference, among other factors.

It was also possible to partially verify, through the application, how peripherals deal with PHY changes. In the application, it was verified the type of PHY used at the moment, being the "LE 2M", with high data transmission rate. Then, the PHY type was changed to "LE 1M", which is the standard type, and the PHY type used at the moment was checked again, noting the change, as shown in figure 22 .

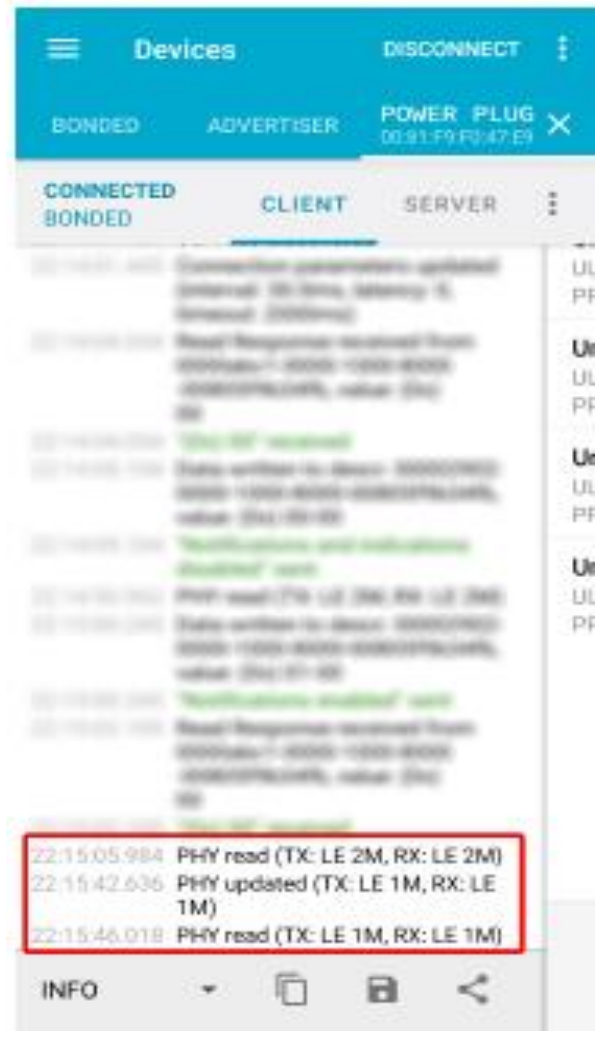

Figure 22. PHY type updating process
To test the operation of the "LE CODED" PHY, the BTOOL software, found in the SimpleLink CC2640R2 SDK 3.30 was used. When starting the software, the option "scan" was selected to start the scan, and immediately afterwards the device was selected, in this case the Gas Sensor peripheral, and the "Establish" button was pressed to start the connection. With the connection established, PHY was updated to "LE CODED" and the current PHY was read. In the connection log it is possible to visualize the use of the PHY "LE CODED", as shown in figure 23.

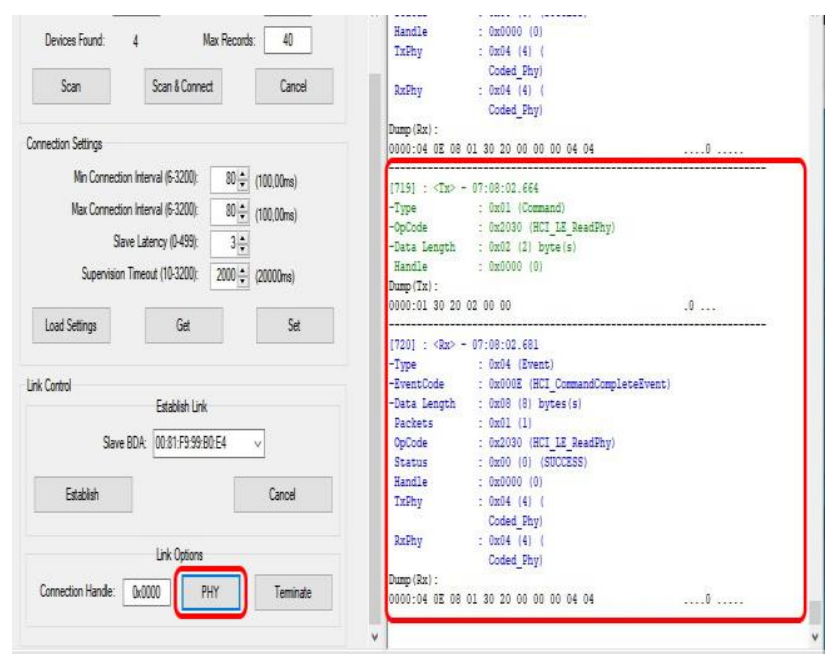

Figure 23. Bluetooth connection using PHY type "LE CODED"

\section{B. Central Hub}

To test the receipt of data from the Firebase database in NodeMCU, the receipt of data from peripherals in LaunchKit, and, consequently, the communication between NodeMCU and LaunchKit, a USB-Serial converter connected to the UART communication transmission lines was used. To view the data, the RealTerm software was used [13].

To perform the test, the Smart Power Plug and Gas Sensor peripherals were connected to the hub. Using the RealTerm software it was possible to see the Smart Power Plug peripheral having its status changed, with the LaunchKit sending the value "0x01" through UART when the Power Plug's relay activates, and sending the value "0x00" when it deactivates. It was also possible to see the LPG Sensor peripheral having its status changed, with the LaunchKit sending the value "0x01" when gas was being detected, and the value "0x00"when the gas was no longer being detected. 


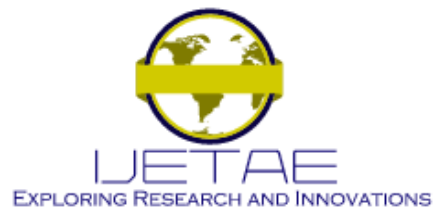

International Journal of Emerging Technology and Advanced Engineering

Website: www.ijetae.com (ISSN 2250-2459, ISO 9001:2008 Certified Journal, Volume 10, Issue 08, August 2020)

For the data transmission done by NodeMCU, when receiving updates from the Firebase database, it was possible to see it sending through UART the value "0x01" and "0x00" referring to the Smart Power Plug peripheral.

During the development of the project, the UART communication presented some problems, due to the fact that there is a transition of states in the pins of RX and TX of both devices, during the initialization of the microcontroller, causing noise in the input buffers, and causing data loss and device out of sync. To solve the problem, in the codes of both devices, a routine was created to clear the input buffers at startup, so that they start synchronized.

\section{Integration}

For the integration test between all the components developed, the test environment was set up as shown in figure 24 .

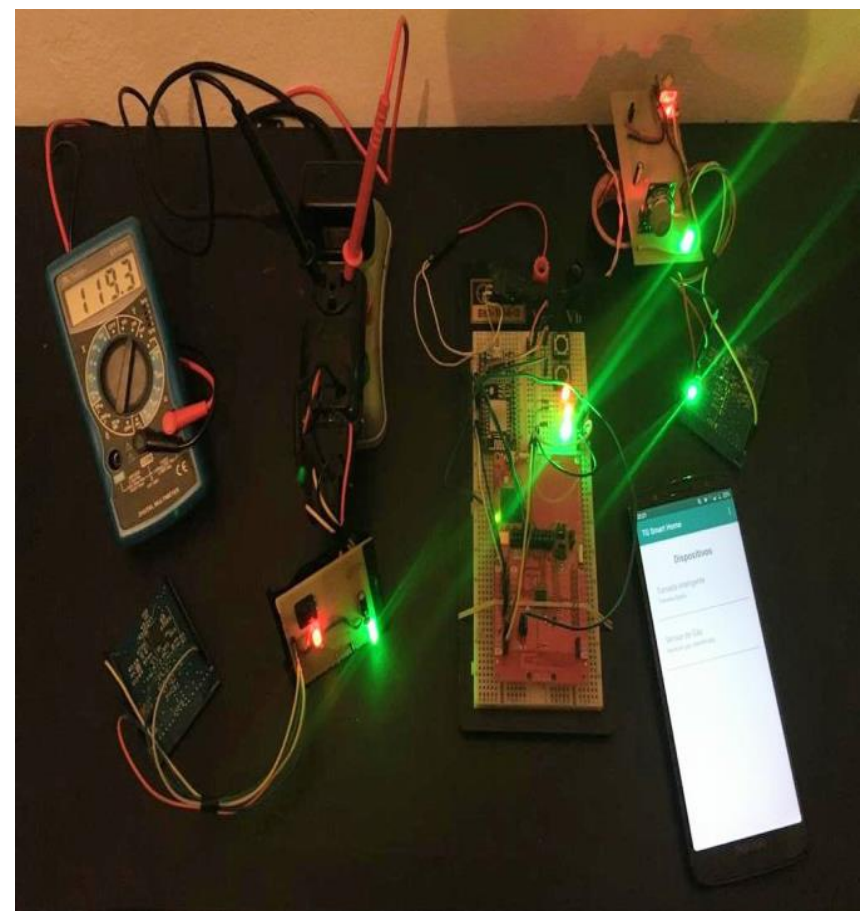

Figure 24. Project test environment

The integration was tested, firstly, by creating a new user in the Android application, pressing the "CADASTRESE" (Register) button on the main screen. After opening the registration screen, all information was filled in, and, to complete the registration, the button "CADASTRAR" (Register) was pressed, sending the information to the Firebase server, as shown in figure 25.
On the registration screen it is also possible press the "CANCELAR" (Cancel) button to return to the main screen.

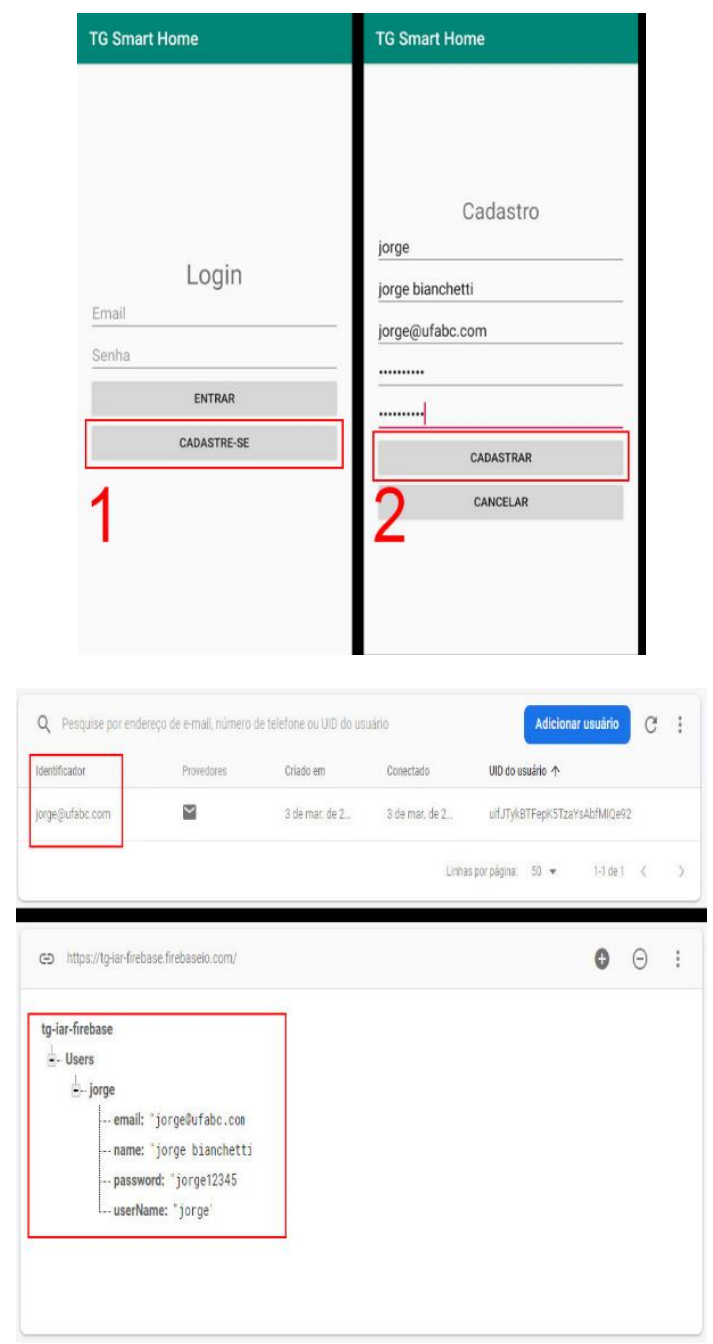

Figure 25. User registration performed in the system integration

After registration is completed, the application returned to the main screen, where it was possible to login using the information entered in the registration and pressing the "ENTRAR" (Login) button. The device view screen was then opened, the menu button was pressed and the option "Adicionar Dispositivo" (Add Device) was chosen, as shown in figure 26. In the menu there is also the option to $\log$ out of the account, pressing the "Sair" (Log Out) button. After opening the device addition screen, the "TOMADA INTELIGENTE" (Smart Power Plug) button was pressed, as shown in figure 27 , to add the device of the same name. 


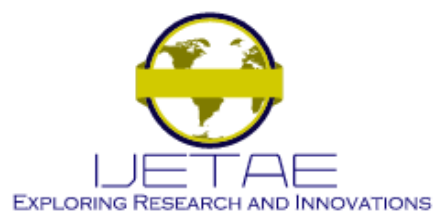

International Journal of Emerging Technology and Advanced Engineering

Website: www.ijetae.com (ISSN 2250-2459, ISO 9001:2008 Certified Journal, Volume 10, Issue 08, August 2020)

The application returned to the device view screen and again the steps in Figure 26 were performed to add the "SENSOR DE GÁS" (Gas Sensor) device.

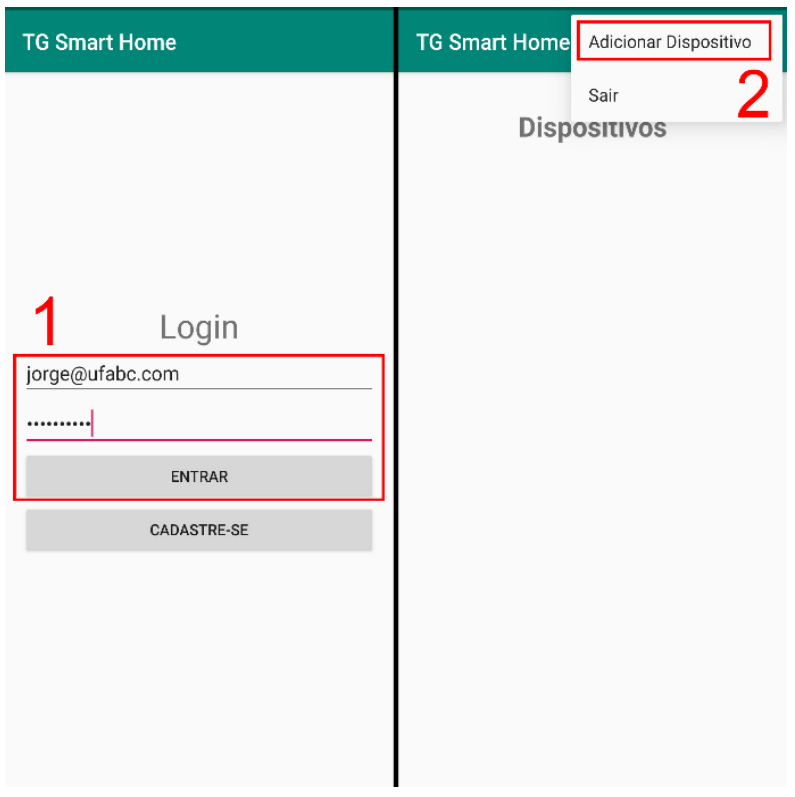

Figure 26. Initiation of the device addition process

When adding the devices by the application, they were also created in the Firebase database, with the respective types, values and keys, as shown in figure 27. Upon returning again to the devices screen, it was possible to verify the addition of the devices by the application.

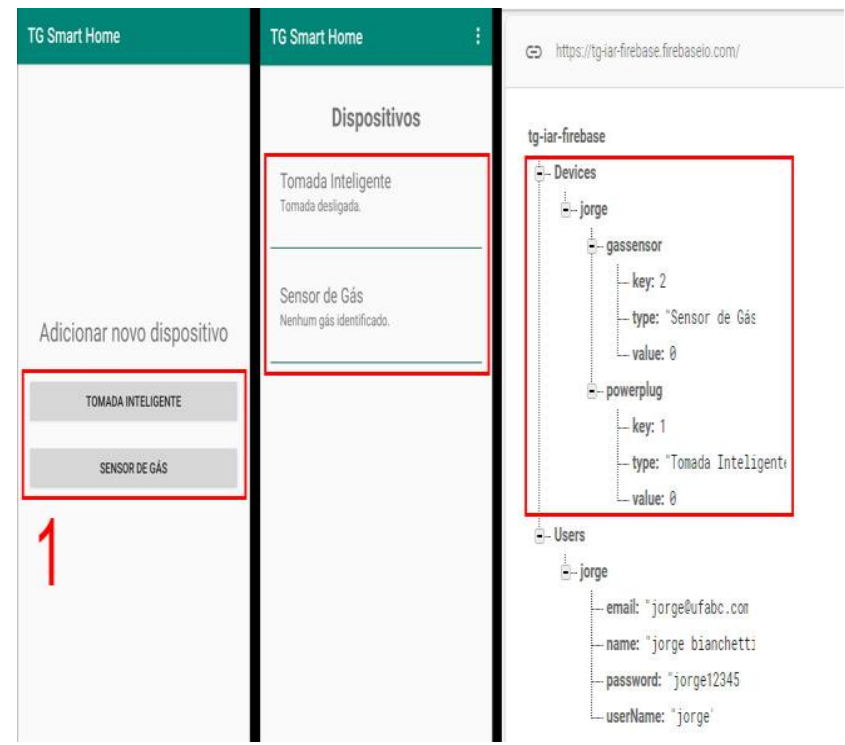

Figure 27. Adding devices to the application and to the Firebase database
With the devices added, the hub was connected to a 5volt power supply, and its green LED lit, indicating the presence of power. A Wi-Fi access point was then generated, as shown in figure 28 , where the data from the Wi-Fi network was entered via the smartphone, in addition to the user name and password previously registered in the Android application. After that, the yellow LED lits, indicating that the connection was successful.

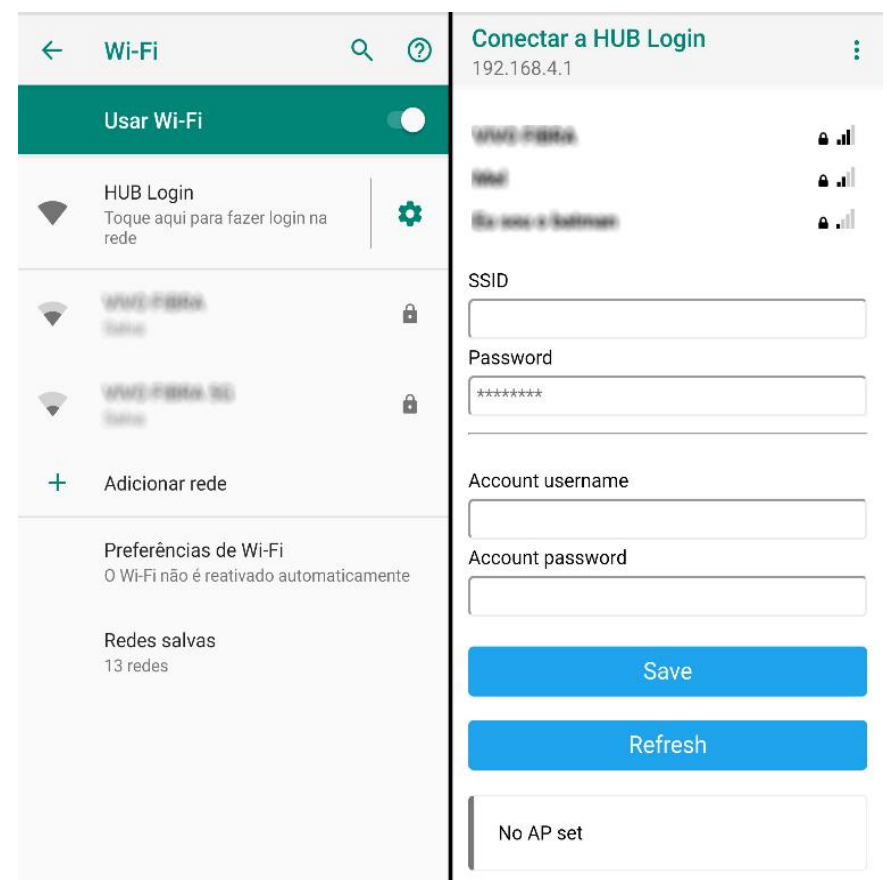

Figure 28. Wi-Fi access point created

Both peripherals were then turned on. In order to connect the peripherals to the hub, the hub connection button was pressed twice, once to add each device, causing the red LED lit, indicating the connection of the peripherals.

After the initial setup, the whole set operation was then tested. To test the LPG Sensor, a lighter was used, which contained butane gas, allowing only its gas to leak. Once this was done, the application showed the Gas Sensor peripheral not detecting the presence of gas, with its red LED off, and the application indicating "Nenhum gás identificado" (No gas identified) message. When the gas was released from the lighter, the peripheral lit the red LED, indicating the presence of the gas, and the application displayed the message "Presença de gás identificada" (Presence of gas identified), as shown in figure 29. 


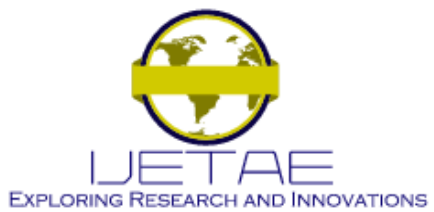

International Journal of Emerging Technology and Advanced Engineering

Website: www.ijetae.com (ISSN 2250-2459, ISO 9001:2008 Certified Journal, Volume 10, Issue 08, August 2020)

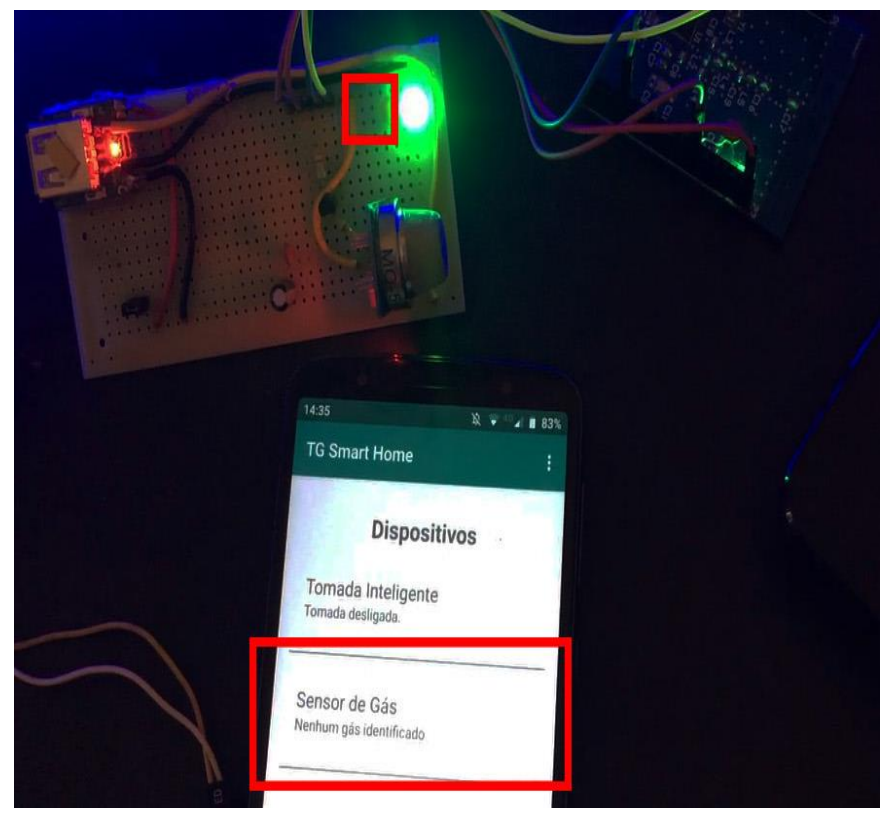

Figure 29 (a). Gas sensor peripheral operation (no gas identified)

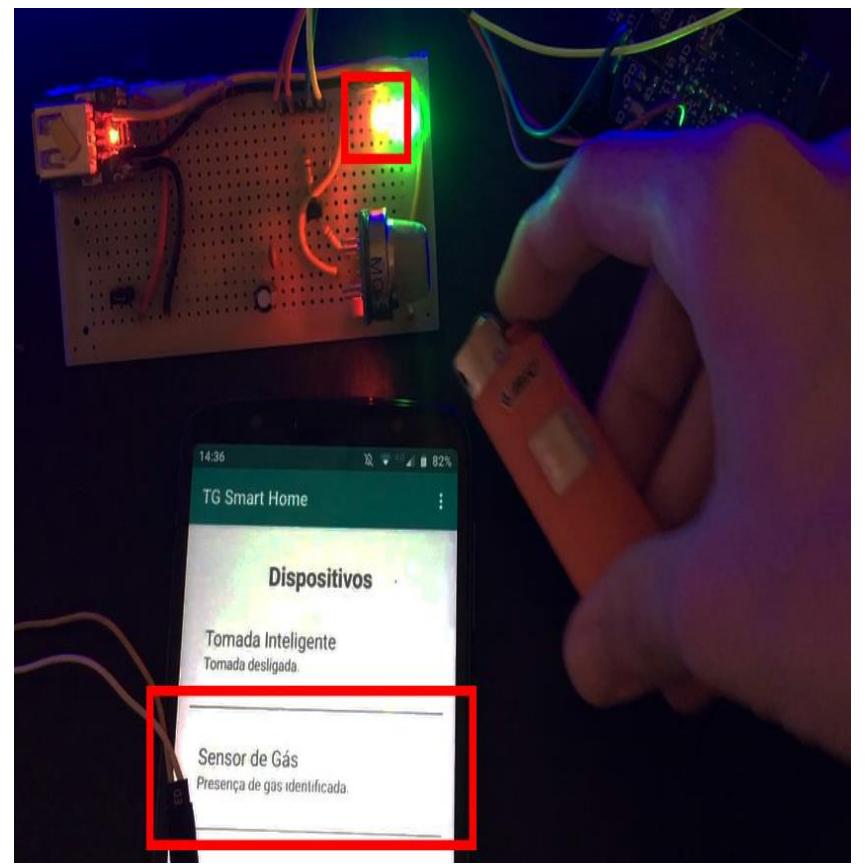

Figure 29 (b). Gas sensor peripheral operation (presence of gas identified)

To test the Smart Power Plug peripheral, a multimeter was used to observe the electrical voltage between the terminals where the device will be connected.
To turn the device connected to the Smart Power Plug on or off, the push button on the board can be used, as well as the Android application, which also shows the status of the Smart Power Plug and the Gas Sensor.

By pressing the button on the Smart Power Plug peripheral board, it is possible to activate and deactivate the relay even without it being connected to the hub. With the Smart Power Plug peripheral connected to the hub, the "TOMADA INTELIGENTE" button in the application was pressed to activate and deactivate the peripheral, as presented in figure 30, showing the application displaying the message "Tomada Ligada" (Power Plug On) and "Tomada Desligada" (Power Plug Off).

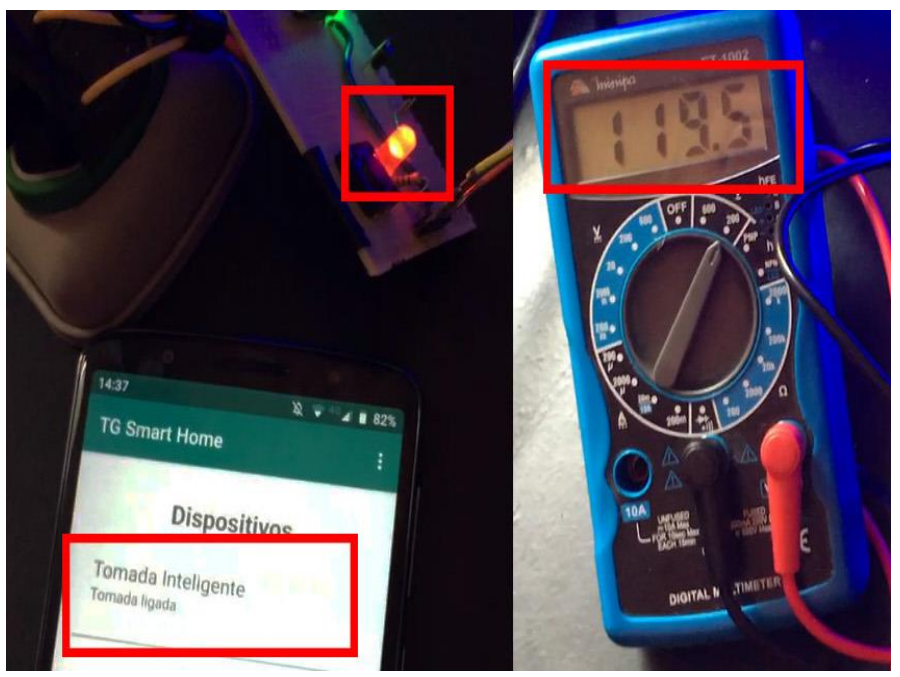

Figure 30 (a). Smart Power Plug peripheral operation (plug on).

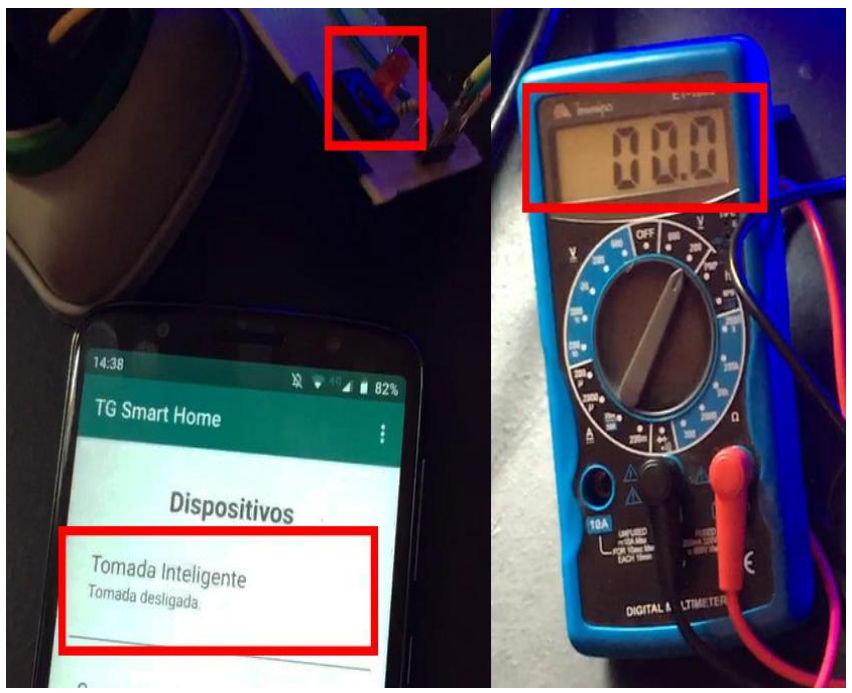

Figure 30 (b). Smart Power Plug peripheral operation (plug off). 


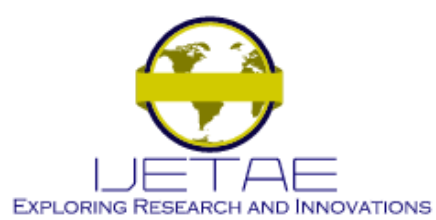

\section{International Journal of Emerging Technology and Advanced Engineering Website: www.ijetae.com (ISSN 2250-2459, ISO 9001:2008 Certified Journal, Volume 10, Issue 08, August 2020)}

When both peripherals were turned off, the red LED on the central hub turns off, indicating that there were no more peripherals connected. It was also tested to reset the settings saved in NodeMCU, by turning off the hub, pressing and holding the reset push button and turning the hub on again. The yellow LED flashed 5 times and again an access point was created for the entry of new information.

For distance testing, the peripherals were installed inside the house, at a distance of approximately 20 meters from the central hub, with a floor and some walls between the two points. Throughout the test, there was not observed malfunction between communications, and the devices operated normally for more than 1 day of uninterrupted use.

\section{CONCLUSIONS}

Home automation systems have been gaining more and more space in the market in recent years, and it is natural to explore new technologies to add new features to the systems.

This paper aimed to describe the development of a home automation system using simple and effective technologies, such as the Firebase real-time database, and technologies that are still little explored in this type of application, such as Bluetooth 5.0. For this purpose, it was developed a central hub, which communicates with two peripheral modules via Bluetooth 5, being them a Smart Power Plug and a LPG Sensor, peripheral that sends and receives data from a Firebase database in the cloud. The objective was successfully achieved.

From this project it was possible to test the operation of the new features implemented in version 5 of the Bluetooth communication protocol, such as the PHYs LE CODED and LE 2M, of high distance and high data rate respectively, using the Texas Instruments CC2640R2F microcontroller. It was also possible to prove the versatility of the Firebase database in real time together with the NodeMCU development kit and an application for the Android platform.

During the development of the project, some difficulties were faced, such as understanding the process of developing an application using a real-time operating system, or RTOS, limitations in the library that allows NodeMCU communication with the Firebase server and noise on the UART interface. Despite these difficulties, the main objective of the work was accomplished.
Although the Bluetooth antenna has performed properly, for a measurement with higher reliability, it is still necessary to carry out procedures in laboratories with appropriate equipment for this purpose.

The sensors used in this experiment worked properly, being properly commanded by the App implemented in the smartphone and firebase, showing promissory results to smart home automation applications.

\section{Acknowledgments}

The authors thank the Federal University of ABC for the support given to this project.

\section{REFERENCES}

[1] Carbou, Romain; Diaz, M.; Exposito, Ernesto; Roman, R. Digital Home Networking, (2013). DOI: 10.1002/9781118602829.

[2] International National Data Corporation. Worldwide Spending on the Internet of Things Will Slow in 2020 Then Return to DoubleDigit Growth, According to a New IDC Spending Guide", in: https://www.idc.com/getdoc.jsp?containerId=prUS46609320, accessed at: 18/07/2020.

[3] Bluetooth SIG. 2020 Bluetooth Market Update, in: https://www.bluetooth.com/bluetooth-resources/2020-bmu/, accessed at: 18/07/2020.

[4] Sánchez, Antonio; Carro, Belén. Digital Services in the 21st Century: A Strategic and Business Perspective. New Jersey: JohnWiley \& Sons, Inc, (2017).

[5] Chew, Daniel. Protocols of theWireless Internet of Things, p. 21-45, ( 2018). DOI: 10.1002/9781119260608.ch2.

[6] Woolley, Martin. Developer Study Guide: An introduction to Bluetooth Low Energy Development. (2017), e-book.

[7] Texas Instruments. SIMPLELINK CC2640R2 SDK BLE5-Stack User's Guide 1.01.06.00", http://dev.ti.com/tirex/content/simplelink_cc2640r2_sdk_3_20_00_2 1/docs/ble5stack/ble_user_guide/html/ble-stack-5.x-guide/indexcc2640.html, accessed at: 04/08/2019.

[8] Woolley, Martin. Bluetooth 5 Go Faster. Go Further, Bluetooth SIG, (2016), 4-25.

[9] Costa, Igor; Araujo, Jean, Dantas, Jamilson, Campos, Eliomar, Silva, Francisco Airton, Maciel, Paulo. Availability Evaluation and Sensitivity Analysis of a Mobile Backend-as-a-service Platform. Quality and Reliability Engineering International, 32, (2015). DOI: 10.1002/qre.1927.

[10] Meier, Reto; Lake, Ian. Professional Android R. (2018). ISBN 9781118949528. DOI: 10.1002/9781119419389.

[11] Paulo, Jean. Desenvolvimento de um aplicativo Android e de uma interface Bluetooth para um dinamômetro biomédico. Doctoral Thesis (2014).

[12] Texas Instruments. SimpleLink CC2640R2 SDK", in: http://www.ti.com/tool/SIMPLELINK-CC2640R2-SDK, accessed at: $20 / 12 / 2019$.

[13] REALTHERM: SERIAL/TCP TERMINAL, version 2.0.0.70, SourceForge, 22/05/2014. Available on: <https://sourceforge.net/projects/realterm/files/Realterm/2.0.0.70/>. 